\title{
On the syntax and semantics of Italian spatial Ps
}

\author{
Francesco-Alessio Ursini \\ Stockholm University \\ francesco.ursini@english.su.se
}

\begin{abstract}
This paper offers a unified approach to Italian spatial prepositions, such as di fronte a 'in front of', verso 'towards', in 'in', dietro a 'behind', and nel mezzo di 'in the middle of'. Three assumptions play a key role. First, Italian spatial prepositions can differ sensibly in their morphological structure, but share the same syntactic properties. Second, their sentential distribution is in part context-sensitive, thus based on the categories with which they combine. Third, their semantic contribution is "layered", in the sense that it includes the meaning dimensions of both aspectual boundedness and specificity. The main result is a generalised theory on the structure and semantic interpretation of these prepositions.
\end{abstract}

Keywords: Italian; adpositions; Distributed Morphology; lexical aspect; Type-Logical Syntax

\section{Introduction}

In recent years, a wealth of studies have focused on the category of spatial Ps ${ }^{1}$ (henceforth SPs) and their cross-linguistic distribution, both from a typological (e.g., Levinson \& Wilkins 2006; LeStrade et al. 2010) and minimalist (generativist) perspective (e.g., Asbury 2008). Within minimalism, works within the cartographic approach also pursue a cross-linguistic analysis (den Dikken 2010; Svenonius 2010), and have offered fine-grained analyses of this category.

Given this wealth of data, one perhaps surprising fact is that few studies investigate Italian spatial prepositions (henceforth: ISPs) in detail. A first partial exception is a group of recent papers that investigate the ability of certain ISPs to include ${ }^{2}$ the affix $a$ in their morphological

\footnotetext{
${ }^{1}$ This label stems from the initial letter of the parts of speech included in this category: Prepositions, Particles, Prefixes, Postpositions (Asbury 2008). We mostly focus on (Italian) prepositions and particle-like elements such as indietro (Rizzi 1988, 510-514). We introduce precise labels in the next section.

${ }^{2}$ In this and the next section, we employ the notion of "co-occurrence" to discuss the distribution of both ISPs and verbs at a pre-theoretical level. We introduce the formal notion of merge in the next section.
} 
structure, which is taken to be a non-spatial, aspectual-like element. When this happens, an ISP can receive a different interpretation than when this affix is absent (Tortora 2005; 2006; 2008; Folli \& Ramchand 2005; Folli 2002; 2008). A second partial exception is Rizzi (1988), a thorough and descriptive work on ISPs that is, however, only accessible to speakers of Italian. Two examples are in (1)-(2), and include the ISPs dietro and dietro alla:

(1) La palla è dietro la scatola.

the ball is behind the box

'The ball is in one place behind the box.'

(2) La palla è dietro alla scatola.

the ball is behind at-the box

'The ball is in some place behind the box.'

When an ISP such as dietro occurs without the affix $a$, it denotes a single, specific position out of several positions that can be defined as "behind" the box. When dietro occurs with $a$, it conflates ${ }^{3}$ with the definite article $l a$ to form alla 'at-the', it denotes any possible location that can be defined as "behind" the box, as the translation 'in some place behind the box' suggests. Works such as Tortora (2008) suggest that this distinction can be reduced to an aspectual difference between "bounded" (dietro) and "unbounded" positions (dietro alla). Although appealing, this analysis of ISPs can only cover a subset of the relevant ISP data. Other ISPs, instead, must or must not co-occur with $a$, regardless of the interpretation at stake. Some examples are in $(3)-(4):^{4}$

(3) Il treno è accanto alla/*la stazione

the train is next at-the $/ *^{*}$ the station

(4) Mario cammina verso la/*alla stazione

Mario walks towards/ towards to-the station

The ISP accanto 'next to', in (3), must co-occur with alla, otherwise the sentence will be ungrammatical; the bounded reading seems favoured, if

${ }^{3}$ Conflation is usually defined as a combination of two morphosyntactic elements into a single "word" (Talmy 2000, ch. 5; Hale \& Keyser 2002, ch. 3). We return to this notion in section 4 .

${ }^{4}$ For reasons of space, we omit translations when the glosses are almost identical to English translations. 
not the only licensed one. The ISP verso cannot co-occur with alla 'at the', and only an unbounded reading can arise. Although some works acknowledge these facts (Tortora 2005; Folli \& Ramchand 2005), they do not investigate the possibility of giving a unified analysis of the distributional properties of all ISPs. Furthermore, although Rizzi (1988) offers a broader descriptive picture, it does not cover this set of data, nor does it attempt to present a putative general account of ISPs. Thus, previous works on ISPs only offer a partial syntactic and semantic analysis of this category. A theoretical alternative could involve the extension of cartographic approaches to these data (e.g., Asbury 2008), but other minimalist alternatives could be equally feasible, too. Therefore, ISPs offer a challenge for any minimalist theory of SPs, cartographic or not.

The main goal in this paper is to ameliorate this dearth of research on ISPs by proposing a novel, unified analysis of this category that is also consistent with previous works. Our plan is as follows: we first offer a broad descriptive overview of the ISPs data in need of an explanation (section 2). We then introduce our formal tools: a combination of Distributed Morphology (henceforth DM, Embick \& Noyer 2006; Harley 2010a;b; 2012), typelogical calculi (Moortgat 2010), and a situation semantics interpretation (Kratzer 2007). Section 3 presents an analysis of the morphological data; section 4 presents an analysis of the semantic data. Section 5 concludes the paper.

\section{Basic notions and the ISPs data}

The goal of this section is to present basic notions about spatial Ps (section 2.1 ), old and novel data, and a set of problems in need of a solution (sections $2.2,2.3$ and 2.4).

\subsection{Basic descriptive notions about spatial Ps}

Our goal in this short section is to introduce key descriptive notions about spatial Ps that we employ in our discussion. It is generally assumed that SPs denote a spatial relation that holds between a landmark object or ground, and a located entity or figure (Talmy 2000, ch. 1). SPs are further distinguished between locative and directional types (e.g., Cresswell 1978; Jackendoff 1983; 1990; Wunderlich 1991; Nam 1995; Zwarts \& Winter 2000; Svenonius 2008). Locative Ps denote the unchanging location over time of a figure with respect to a ground (e.g., English in, at, in front of, 
behind). Directional Ps denote the direction or "path" that a figure follows when moving with respect to a ground (e.g., to, from, through). Locative Ps can be further distinguished between projective Ps and non-projective Ps. Projective Ps denote a relation between figure and ground specified along an axis or projection (e.g., in front of). Non-projective Ps denote a relation expressing only topological information (e.g., in). Consider examples (5)-(7), in which Mario and the garden, respectively, are the figure and ground DPs:

(5) Mario sits in the garden

(6) Mario sits in front of the garden

(7) Mario has gone to the garden (locative, non-projective P: in)

(locative, projective P: in front of )

(directional P: to)

A precis on the taxonomy presented in (5)-(7), and its application to ISPs data, is due. Italian is usually considered a "verb-framed" language (Talmy 2000, ch. 5; Folli 2008). Most verbs can be distinguished as having either a locative or a directional sense, while ISPs are inherently ambiguous and receive their interpretation based on the verb they co-occur. Thus, the "locative" copula $\grave{e}$ 'is' in (1)-(4) determines a locative interpretation for dietro 'behind'and accanto 'next to'; the "directional" cammina 'walks' determines a directional interpretation for verso 'towards' (Folli \& Ramchand 2005; Folli 2008). This phenomenon seems not to be limited to Italian: two English equivalent examples are the boys walk towards the station and the office is towards the station. For the sake of clarity, though, we will mostly discuss the phenomenon as it applies to ISP data. ${ }^{5}$

For these reasons, we employ the following two assumptions in our analysis of ISPs. First, we assume that all ISPs are lexically underspecified (i.e., ambiguous) with respect to this dimension of meaning (Harbour 2007; Egg 2011). Second, we employ three descriptive, theory-neutral labels. A first is "projective ISP" for morphemes such as dietro 'behind' or accanto 'next to' that denote axial/projective components of meaning (cf. also Svenonius 2006; 2010; Pantcheva 2008; Terzi 2010). A second is "simple ISP" for the non-projective affixes $a$ 'at/to', $d i$ 'of'. A third is "complex ISP", for ISPs such as dietro alla 'behind some location' and di fronte alla 'in front of some location'. We make this choice to have theory-neutral terms at our disposal, in our analysis of previous data and proposals, and novel data.

\footnotetext{
${ }^{5}$ Thanks to an anonymous reader for suggesting this formulation of the problem.
} 


\subsection{The basic ISP data: Rizzi (1988)}

The goal in this section is to give an overview of the data in Rizzi (1988). Given the wealth of data that we discuss, we divide the section in three thematic sub-sections.

\subsubsection{Simple ISPs: Syntactic and semantic properties}

The goal of this section is to discuss the syntactic and semantic properties of simple ISPs, called preposizioni primarie 'primary prepositions' in Rizzi (1988). These are defined as syntactic heads that must take a complement DP in order to be syntactically well formed. Complex ISPs are defined as ISPs that may occur without a complement DP and can thus be subject to argument demotion (den Dikken 2006, ch. 2; Svenonius 2010), hence appearing to have an adverbial-like distribution. Consider (8)-(11):

(8) Mario è al tavolo da biliardo.

Mario is at-the billiard pool

(9) *Mario é a(l tavolo da biliardo).

Mario is at(-the billiard pool)

(10) Mario é dietro (al tavolo da biliardo).

Mario is behind (at-the billiard pool)

(11) Luigi é di fronte al tavolo da biliardo.

Luigi is in front at-the billiard pool

Mario é dietro (al tavolo da biliardo)

Mario is behind (at-the billiard pool)

As (8)-(9) show, simple ISPs such as $a$ 'at/to' cannot undergo object demotion. However, when argument demotion can be identified as form of an ellipsis (Merchant 2001; 2004) and the demoted ground DP is anaphorically related (identical, in (11)) to the previous ground DP, demotion is licensed. This happens when complex ISPs such as dietro alla 'behind at-the/to-the' is involved (viz. (10)-(11)), but not when simples ISPs are involved (viz. $a$ 'at' in (8)-(9)). When this happens, as (10)-(11) show, the optional $\mathrm{P}$ alla is demoted together with the argument DP (here, tavolo da biliardo 'pool table').

Furthermore, as the data in (1)-(4) and (8)-(11) show, the P a must conflate with the definite article, when a definite article immediately follows this ISP. The same process of conflation occurs with other simple 
Ps, except for tra/fra 'between' and per 'for'), at least in the orthographic norm. Before we continue our discussion, we address this phenomenon. This phenomenon is labelled as raddoppiamento sintattico, or "syntactic doubling" in English (henceforth RS, Napoli \& Nevis 1987). RS can be defined as the "doubling" of the consonant at the beginning of a word, when this word is the right-branching constituent of a phonological word, and the left-branching constituent ends with an unaccented vowel. The result is the apparent "doubling" of the initial consonant. From $a$ (a P head) and, for example, la (a D head), we have $a-l-l a$, a conflation of two heads mediated via the "doubled" consonant $-l$ -

The theoretical status of RS is controversial, in particular its relation to the notion of conflation (Frascarelli 2000; Hale \& Keyser 2002, ch. 3; Absalom \& Hajek 2006). However, its relevance for our data and analysis seems clear, hence we will address RS and its relation to syntactic processes, in our analysis. For the moment, we present Rizzi's (1988) list of simple ISPs:

(12) Simple ISPs $=\{a$ 'at/to', $d a$ 'from', $d i$ 'of', in 'in', tra/fra 'between', su 'on/to' $\}$

$a$, $d a$ and $d i$ can also occur as non-spatial ISPs. The first three simple ISPs can co-occur with other projective terms, such as dietro 'dietro' or accanto 'next to', to form complex ISPs (i.e., $a, d a$ and $d i$ ), which offers an aspectual contribution to interpretation. As the translations suggest, simple ISPs can be ambiguous between a locative and directional reading (e.g., $a$ 'at/to'), although one of the two readings can be fairly restricted. As Rizzi (1988) does not discuss this semantic ambiguity of simple ISPs, we procrastinate a more precise discussion to section 2.3 .

\subsubsection{Complex ISPs: Morphosyntactic and semantic properties}

The goal of this section is to offer an analysis of complex ISPs (preposizioni secondarie or preposizioni avverbiali ('secondary' or 'adverbial prepositions' in Rizzi 1988, §3.1). Most of these ISPs involve a projective ISP, e.g., dietro 'behind', which can co-occur with a simple ISP, e.g., a. Complex ISPs are divided into three classes: ISPs that must include a simple ISP in order to be grammatical (class I), ISPs that may do so (class II), and ISPs that cannot co-occur with a simple P (class III). Key examples are in (13)-(15):

(13) Class I

Mario è davanti al $/ *_{\text {il }} \quad$ tavolo da biliardo.

Mario is in front at-the $/ *(\mathrm{P})$-the billiard pool 
(14) Class II

La lampada è sopra al/il tavolo da biliardo.

the lamp is above at-the/(P)-the billiard pool

(15) Class III

Mario si siede verso *alla/la macchina.

Mario REFL sits towards *at-the/(P)-the car

In (13), the omission of a from davanti alla 'in front of-the' renders the sentence ungrammatical (cf. (13)). In (14), sopra 'above' can freely cooccur with $a$, with a slight difference in interpretation: sopra al tavolo 'above the table' denotes an unspecified vertical, positive position, sopra $i l$ tavolo a specific one. In (13)-(14), we assume that a phonologically null "(P)" head is also present, licensing the relevant bounded representation via conflation with the definite article. ISPs such as verso 'towards' cannot cooccur with a simple $\mathrm{P}$ such as $a$, regardless of the interpretation (cf. (15)). The three relevant, near-exhaustive lists of such examples are illustrated in $(16)-(18)$ :

(16) Class I = \{ accanto 'beside', addosso 'against', davanti 'ahead of', dirimpetto 'opposite' , incontro 'towards', intorno/attorno 'around', vicino 'near'

(17) Class II = \{attraverso 'through', dentro 'inside', dietro 'behind', lungo 'along', oltre 'past', presso 'close to', rasente 'over', sopra 'above'\}

(18) Class III $=\{$ verso 'towards' $\}$

As the lists suggest and as discussed in passing in Rizzi (1988), several projective ISPs seem to involve the conflation of at least two morphemes. The alternation between the two varieties intorno/atttorno 'around', but also accanto 'next to' and addosso 'against', may be traced to their Latin ancestors (e.g., ad-and tornium for attorno; intorno is another possible form). Similar patterns have been attested in Spanish (Fábregas 2007; Ursini 2013; 2015), English (Svenonius 2010; Ursini \& Akagi 2013b) or Persian (Pantcheva 2008). These ISPs are are ambiguous between a locative and a directional reading, for the most part, but we procrastinate a discussion of this fact to section 2.4. As Rizzi (1988) discusses, the simple ISP that co-occurs with Class I and II ISPs is a 'at/to'. Hence, the partition of complex ISPs into three classes seems justified, given these sets of data. 


\subsubsection{Complex ISPs: Syntactic problems}

The goal of this section is to present a broader set of syntactic properties of complex ISPs, and three problems they pose for Rizzi's taxonomy.

First, when a ground DP is a pronoun, $d i$ 'of' must occur with an ISP, regardless of its class. Thus, with respect to these specific types of ground DPs the distinction among three complex ISP classes seems vacuous, as shown in (19)-(21):

(19) Mario è dietro di $/ *(\mathrm{P})$ lui.

Mario is behind of $/ *(\mathrm{P})$ him

(20) Mario è accanto a/*di lui.

Mario is beside to $/{ }^{*}$ of him

(21) Mario va verso di $/ *(\mathrm{P})$ lui.

Mario goes towards of $/ *(\mathrm{P})$ him

Examples (19)-(21) show that when a ground DP is a pronoun, then $d i$ is inserted between a projective ISP and the DP, regardless of the ISP class. Other types of pronouns cannot co-occur with $d i$ 'of', and must co-occur with a $\mathrm{P}$ directly (e.g., demonstratives: verso quello vs. ${ }^{*}$ verso di quello, 'towards that').

A second problem involves a su-set of data discussed in Rizzi (1988), and DPs acting as grounds in coordinated and disjunct phrases. When at least one conjunct is a pronoun, and the other is (possibly) an R-expression, $d i$ insertion is blocked. The only exception is when both co-occur with distinct instances of $d i .{ }^{6}$ Consider (22)-(23):

(22) Mario va verso *di me o lui/di me o di lui.

Mario goes towards * of me or him/of me or of him

(23) Mario siede dietro *di me e Luigi/di me e di Luigi.

Mario sits behind $*_{\text {of }}$ me and Luigi/of me and of Luigi

Thus, this insertion rule seems sensible to the fact that the two ground DPs are introduced via coordination/disjunction, and a specific ISP. As in the case of the first set of data, these patterns suggest that a sharp taxonomy for complex ISPs is not so strongly motivated when discussing a broader set of data.

${ }^{6}$ I thank an anonymous reviewer for suggesting this much more precise analysis of the data. 
A third problem concerns the possibility to front an ISP in a sentenceinitial position, via a specific form of locative inversion (den Dikken 2006, ch. 1; Svenonius 2010). Rizzi (1988) discusses one case, involving ground DPs and simple ISPs forming a distinct phrase, the simple ISP obligatorily being $a$ 'at/to'. Consider (24)-(26):

(24) Mario siede dietro di lui.

Mario sits behind of him

(25)*Di lui/A lui/A Luigi, Mario siede dietro

of him, Mario sits behind

(26) Q: Mario siede di fronte alla macchina?

Mario sits in front of the car

A: Alla casa, Mario siede di fronte.

at-the house Mario sits of front

In (24)-(26), inversion/extraction always involves the obligatory realisation of $a$ 'at/to', which must appear in a displaced position with the ground DP. These sentences are usually acceptable when they act as contrastive topics, have a rising intonation contour, or appear in contrastive answers (Krifka 2001). For instance, a speaker can ask whether Mario is sitting in front of the car, and obtain a contrastive answer, as in (26). In this case, alla casa 'at the house' denotes that the house is the ground, instead of the car. Overall, this and the first two sets of data suggest that the taxonomy proposed in Rizzi (1988), although accurate for unmarked distribution patterns of these ISPs, becomes less adequate for other patterns. Given the context-sensitivity of simple ISPs as the "main" heads, a more flexible categorization seems a more adequate approach.

\subsection{Basic data: The novel data}

The goal of this section is to discuss in more detail a group of seemingly heterogeneous data only marginally discussed in previous accounts: semantic underspecification; inherent semantic ambiguity; the semantics of a class of ISPs that we dub "multi-morphemic" ISPs; and a wider set of locative inversion data.

\subsubsection{Novel ISPs data: Semantic underspecification}

As we discussed so far, all ISPs seem to be underspecified, depending on the verb's interpretation as "directional" or "locative". When ISPs receive a 
directional interpretation, the presence of optional $a$ 'at/to' can discriminate between a directed and a "located" motion interpretation. In the latter case, the verb only denotes the manner by which a certain event of motion occurs, not its direction (Folli 2002; 2008).

A first set of data that these works do not discuss, however, pertains to whether this pattern holds for all ISPs, given the flexible nature of $a$ 's distribution. Since the distribution of this simple ISP with projective ISPs determines the aspectual reading of a resulting complex ISP, one would predict that the distribution of verbs would be influenced accordingly. The presence of $a$ would consequently determine whether the lexical aspect reading of a sentence is bounded or unbounded. Consider thus (27)-(31):

(27) Mario corre al tavolo da biliardo (in un minuto/*per un minuto). Mario runs at-the billiard pool (in one minute $/ *^{\text {for }}$ one minute)

(28) Mario va dietro al/il divano (in un attimo/*per un attimo)

Mario goes behind at-the/(P)-the couch (in one moment/*for one moment)

(29) Mario cammina sotto al/il ponte (*in un'ora/per un'ora).

Mario walks under at-the/(P)-the bridge $\left(*_{\text {in }}\right.$ one hour/for one hour)

(30) Mario cammina verso il fiume (*in un'ora/per un'ora).

Mario walks towards the river $(*$ in one hour/for one hour)

(31) Mario cammina davanti alla macchina (*in un'ora/per un'ora).

Mario walks ahead at-the car $\quad\left({ }^{*}\right.$ in one hour/for one hour)

According to the standard diagnostics for temporal adverbs (Dowty 1989; Zwarts 2005; 2008), bounded/telic predicates combine with the temporal adverb in un'ora 'in one hour', and unbounded/atelic ones with per un'ora 'for one hour'. Although Fong (1997) and Zwarts (2005; 2008) discuss in detail how (English) directional spatial Ps can denote bounded, unbounded or underspecified predicates, they also observe that locative spatial Ps invariably denote unbounded predicates. For our purposes, then, we take a coarse-grained stance, and assume that locative readings can be identified with unbounded readings (and vice versa), while bounded readings can be identified with directional readings. Since ISPs are inherently underspecified, this coarse-grained analysis does not apparently risk being too coarse-grained, with respect to the data.

Consequently, if ISPs are underspecified, then any (lexical aspect) type of verb can occur with any ISP, as the occurrence of al 'at-the' with unbounded corre 'runs' in (27) shows. The resulting VP, Mario corre al 
tavolo da biliardo 'Mario runs to the billiard pool', can receive a bounded interpretation, via the semantic contribution of the ISP phrase. Examples (28)-(31) show that other complex ISPs can also contribute to a bounded (viz. (27)-(28)) or an unbounded (viz. (29)-(31)) interpretation, whether they co-occur with $a$ or not. This occurs when these ISPs combine with aspectually unbounded verbs, va 'goes' and cammina 'walk' respectively. The resulting VP can then receive a bounded or unbounded interpretation, depending on the combination of verb and ISP phrase, and the bounded/unbounded reading of an ISP phrase. For instance, we have the unbounded VP Mario cammina davanti alla macchina 'Mario walks in front of the car' in (31), since it only combines with per un'ora 'for one hour'.

In such cases, the presence or absence of $a$ seems to determine a value belonging to a different meaning dimension, that of specificity. Specificity is usually defined as the ability of a DP to denote a unique referent in discourse, known to hearer and speaker (von Heusinger 2012). We make this suggestion, since our cases involve the presence of a definite article, which involves the distinct semantic dimensions of definiteness and specificity (Heim 2012). As our examples and their glosses suggest, $a$ 'at/to' marks the location that a figure occupies (or moves to) as being specific or non-specific. Crucially, specificity on ISPs is defined with respect to the figure's location (viz. (3)-(4)), while ground DPs can involve definite and specific readings. In both cases, the simple ISP a seems not to play a role, with respect to lexical aspect.

\subsubsection{Novel ISP data: The fine-grained interpretation of simple ISPs}

A second set of understudied data pertains to the distribution and interpretation of simple ISPs. One case is $d a$ 'from', which can be underspecified between a locative and directional interpretation. Furthermore, da must co-occur with lontano 'far' and with animate ground DPs, and can be underspecified. The relevant examples are in (32)-(36):

(32) Mario è da Luigi.

Mario is from Luigi

(33) Mario va/viene da Luigi.

Mario goes to/comes from Luigi

(34)*Mario va a Luigi.

Mario goes at Luigi 
(35) Mario è lontano dal biliardo.

Mario is far from-the billiard pool

(36) Mario è fuori di casa.

Mario is out of home

In (32), the intended interpretation is that Mario is located at Luigi's place, with Luigi indirectly acting as the ground entity. In (33), the interpretation of $d a$ depends on the interpretation of the verb, although $d a$ must occur with animate ground DPs (viz. the ungrammatical (34), with a 'to'). Its interpretation corresponds to English 'from' when it co-occurs with viene 'comes', and to 'to' when it co-occurs with $v a$ 'goes'. In (35), da co-occurs with the projective ISP lontano 'far', although the spatial relation under discussion is a locative one. When fuori 'out' is selected, $d i$ 'of' must cooccur with fuori. Thus, $d a$ and $d i$ ae also possible ISP heads, aside $a$ and $d i$.

\subsubsection{Novel ISPs data: Multi-morphemic ISPs and their properties}

A third set of understudied data involves a subset of ISPs that are known as locuzioni preposizionali 'prepositional locutions' (Rizzi 1988, 530-532). These ISPs involve two simple ISPs with a projective ISP between the two simple ISPs. Thus, we label these as "multi-morphemic ISPs", given their morphological structure. Consider (37)-(39):

(37) Mario siede di fronte alla/*la scrivania.

Mario sits of front at-the $/{ }^{*}(\mathrm{P})$-the desk

(38) Mario siede a destra/sinistra della/*la scrivania

Mario sits at right/left of-the/*(P)-the desk

(39) Mario siede nel mezzo della stanza

Mario sits in-the middle of-the room

(40) Multi-Morphemic ISPs $=\{d i$ fronte $a$ 'in front of', a sinistra di to the left of', a destra $d i$ 'to the right of', in cima a 'on top of', in fondo a 'at the bottom of', a Sud di 'South of', a Nord di 'to the North of', nel mezzo di 'in the middle of'\}

In (40), we also have a non-exhaustive list. The examples in (37)-(39) also show that certain multi-morphemic ISPs appear to involve nonprepositional elements in their structure. The ISP nel mezzo di in the middle of', in (39), includes a definite article that conflates with the "first" 
simple $\mathrm{P}$, forming the "complex" $\mathrm{P}$ nel 'in-the', from in-il. ${ }^{7}$ As the data suggest, the "lower" ISP must be realised, otherwise the resulting sentence will be ungrammatical. Note that simple ISPs can appear either "above" or "below" elements denoting axial or projective content, such as fronte 'front' or sinistra 'left'.

\subsubsection{Novel ISP data: A broader view on locative inversion}

A fourth set pertains to the ability of full ISPs to participate in locative inversion. The extraction data discussed in (24)-(26) suggest that ISPs may partially occur in the inverted position, since the "lower" simple ISP and the ground DP can occur in the sentence-initial position. In the case of multi-morphemic ISPs, only "full" ISPs can be inverted: no other intermediate constituents can be inverted. This is shown in (41)-(43):

(41) Di fronte alla finestra, I ragazzi bevono birra.

of front at-the window the boys drink beer

(42)*Fronte alla stazione, Mario fuma una sigaretta di.

front at-the station, Mario smokes a cigarette of

(43) Dietro (alla/la stazione), Mario fuma una sigaretta.

behind (at-the/the station) Mario smokes a cigarette

Thus, only full phrases such as di fronte alla finestra 'in front of the window' can occur in an inverted position. For the sub-type of complex ISPs, this entails that the "higher" simple ISP cannot occur in a sentence-final position, isolated: *fronte alla stazione 'front of the station' is ungrammatical, as (42) shows. Note that inverted ISPs can also undergo argument demotion. Hence, the demoted DP alla stazione in (43) is understood as having been introduced in previous discourse, here omitted for space reasons.

Overall, these and the previously discussed data suggest that ISPs present both syntactic and semantic challenges. ISPs involve a more complex structure than the one suggested in previous works. Furthermore, lexical aspect and specificity, two distinct semantic notions, play a role in the distribution of ISPs, and seem to determine which simple ISP ( $a$ or $d i)$ that can occur in a certain morphosyntactic context.

${ }^{7}$ Depending on whether the projective $\mathrm{P}$ has masculine and or feminine gender features, the article displays agreement morphology with these features. We have nel mezzo di 'in the middle of', in which mezzo 'middle' and $i l$ the' are labelled as masculine gender terms, and alla fine di 'at the end of', in which fine 'end' and la 'the' are labelled as a feminine gender terms. This aspect is immaterial here. 


\subsection{Previous analyses and four problems about ISPs in need of a solution}

The goal of this section is to discuss previous analyses of ISPs (sections 2.4.1 and 2.4.2), and outline four empirical problems in need of a solution (section 2.4.3).

\subsubsection{Previous analyses: Works on ISPs}

In the introduction we discussed recent works that have offered an analysis of some ISPs and their morphosyntactic properties (Tortora 2005; 2006; 2008; Folli \& Ramchand 2005; Folli 2008). These works only focus on a subset of our Class I ISPs, and suggest that $a$ denotes an unbounded location. Consider (44), as a further example:

(44) Mario è accanto al/il tavolo da biliardo.

Mario is next at-the/(P)-the billiard pool

According to Tortora (2008), locative ISPs include one position, called Asp(ect), which determines whether an ISP is "bounded" or "unbounded". While this position is higher than the position associated to the other ISP (here, accanto 'next to'), overt movement results in the linear order observed in our examples, viz. (45) (ibid., 283-284).

(45) a. before movement:

[CP (P) [AspP a [FP (P) [Place accanto [DP il tavolo da biliardo]]]]]

b. after movement:

$\left[\mathrm{CP}(\mathrm{P})\left[_{\text {AspP }}\left[\right.\right.\right.$ accanto $_{j}$ a [FP $(\mathrm{P})\left[\right.$ [elace $t_{j}$ [DP il tavolo da biliardo]]]]]

The structures in $(45 \mathrm{a}-\mathrm{b})$ contains some simplifications, although we represent the silent positions/heads $\mathrm{C}$ (lause) and $\mathrm{F}$, a non-specified functional element. The Place element accanto 'next to' moves into the specifier position of the head Asp, as projected by a: the linear order accanto al tavolo da biliardo 'next to the billiard pool' is obtained.

On the other hand, Folli (2008, 202-203) offers two closely related, movement-free analyses. One involves the standard Path and Place heads (e.g., Kracht 2002; 2004); the other involves two general heads, "R" and "P". These are shown in (46):

(46) a. [Path accanto [Place a- [DP -1 tavolo da biliardo]]]

b. [Path accanto [Place (P) [DP il tavolo da biliardo]]]

c. [PP accanto [RP (R) [DP il tavolo da biliardo]]] 
In addition, a crucial difference between accanto and accanto al lies in that the first ISP includes a silent Place head, unlike the second ISP. Since this work considers ISPs as inherently locative, it treats projective ISPs as elements projecting a Path head. The alternative analysis in (46c), then, generalises the structure of spatial Ps to any $\mathrm{P}$, by involving the two "abstract" heads P and R.

Although appealing, both proposals face three non-trivial problems, when one tries to apply their analysis to our full set of data. First, both approaches seem to make novel, but not necessarily accurate assumptions about the structure of ISPs. One is accanto 'next' being a Path head, rather than a Place head (Folli 2008). Another is the existence of Asp and F heads (Tortora 2008), given that the evidence motivating these assumptions (i.e., $a$ being an aspectual marker) turns out not to be compelling. Second, both proposals would face further problems when applied to multi-morphemic ISPs. While Folli's analysis would perhaps require more positions, Tortora's analysis would need to account why $d i$ in di fronte $a$ 'in front of', but $a$ in a destra di 'to the right of' seem to project a $\mathrm{C}$ head. Third, the distribution of $a$ as a lexical aspect marker would require a different analysis, as this simple ISP is akin to a specificity marker.

\subsubsection{Previous analyses: Cartographic approaches for ISPS}

In this section, we discuss earlier cartographic approaches (Svenonius 2006; Asbury 2008), although our observations could also extend to more innovative proposals (den Dikken 2010; Svenonius 2008; 2010). Cartographic approaches assume that the fine-grained structure of SPs decomposes into several heads, one per distinguishable morpheme in an SP. These head can in turn form an "SP field", a sequence of functional heads arranged in hierarchical order. At least two new heads have been suggested as part of the SP field: Axpart for projective morphemes (e.g., front), and Kase, for functional morphemes such as of. Some examples are in (47)-(48):

(47) [Path (to) [Place in [Axpart front [Kase of [DP the house]]]]]

(cf. Asbury 2008, ch. 2)

(48) [Path (P) [Place di [Axpart fronte [Kase a- [DP -lla casa]]I]]

We represent the Path head via the optional morpheme "(to)", which may be used in examples such as Mario goes (to) in front of the house (Svenonius 2010, 72-76). Its approximate Italian counterpart is in (48), taken from Mario va $(P)$ di fronte alla casa 'Mario goes in front of the house'. Thus, both SPs can be assigned approximately the same structure, but with two provisos. First, related lexical items such as $d i$ and of may occupy different 
positions in the SP field (Place for $d i$, Kase for of). Second, Italian ISPs can involve RS; hence, the distinction between the SP field and the DP field may be blurred (cf. Asbury 2008, ch. 2). Nevertheless, an initial analysis is certainly possible.

Another argument in favour of the cartographic approach is the existence of compositional analyses of the semantics of SPs, based on standard model-theoretic analyses. These analyses assume that Place heads denote region- or vector-like objects, while Path heads denote path-like objects (Zwarts \& Winter 2000; Kracht 2008). A proposal that connects these two analyses is Svenonius (2008), partly presented in (49):

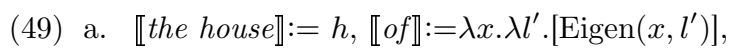
$\llbracket$ of the house $\rrbracket:=\lambda x \cdot \lambda l^{\prime} \cdot\left[\operatorname{Eigen}\left(x, l^{\prime}\right)\right](h)=\lambda l^{\prime}$. $\left[\operatorname{Eigen}\left(h, l^{\prime}\right)\right]$

b. 【front $\rrbracket:=\lambda P . \lambda l . \exists l^{\prime}\left[\right.$ front-part $\left.\left(l, l^{\prime}\right) \wedge P\left(l^{\prime}\right)\right]$, $\llbracket$ front of the house $\rrbracket:=\lambda P . \lambda l \cdot \exists l^{\prime}\left[\right.$ front-part $\left.\left(l, l^{\prime}\right) \wedge P\left(l^{\prime}\right)\right]\left(\lambda l^{\prime} .\left[\operatorname{Eigen}\left(h, l^{\prime}\right)\right]\right)=$ $=\exists l^{\prime}\left[\right.$ front-part $\left.\left(l, l^{\prime}\right) \wedge \operatorname{Eigen}\left(h, l^{\prime}\right)\right]$

c. $\llbracket i n \rrbracket:=\lambda P . \lambda V \cdot \exists l^{\prime}\left[\operatorname{project-out}\left(V, l^{\prime}\right) \wedge P\left(l^{\prime}\right)\right]$, $\llbracket$ in front of the house $\rrbracket:=\lambda P . \lambda V . \exists l^{\prime}\left[\operatorname{project}\left(V, l^{\prime}\right) \wedge P\left(l^{\prime}\right)\right]\left(\exists l^{\prime}\left[\operatorname{front}-\operatorname{part}\left(l, l^{\prime}\right) \wedge\right.\right.$ $\left.\left.\operatorname{Eigen}\left(h, l^{\prime}\right)\right]\right)=\lambda V . \exists l \exists l^{\prime}\left[\operatorname{project}\left(V, l^{\prime}\right) \wedge\right.$ front-part $\left.\left(l, l^{\prime}\right) \wedge \operatorname{Eigen}\left(h, l^{\prime}\right)\right]$

In words, the interpretations of the house and of combine together, in (49a), to derive the interpretation for of the house: the region of space that the house occupies (its "Eigenspace": Wunderlich 1991). This interpretation becomes conjoined with that of front in (49b) and with that of in in (49c): a relation between a house's location $l^{\prime}$ and its front part $l$ is established, defined with respect to a set of vectors directed outside the house. Thus, the interpretation of in front of the house can be compositionally derived.

Overall, although appealing, this analysis does not motivate the proposed logical translations, and lacks a type a type-driven translation, but certainly sketches a compositional account of SPs. Furthermore, its extension to ISPs seems to have at least two problems. First, one has to motivate on a case-by-case basis the exact translation of simple ISPs such as $d i$ and $a$, as they can occur both in the Place and Kase position. Second, the proposal must also be enriched with a treatment of lexical aspect (boundedness) and specificity: the semantics of alla 'at-the' appears beyond the reach of this analysis. With these observations in mind, we summarize our discussion. 


\subsubsection{Interim summary}

We can now summarize, and individuate four ISP problems in need of a solution that have emerged from our discussion of the data, and of previous works on ISPs.

First, the bi-partite analysis of Folli (2008) and cartographic approaches (including the related Tortora 2008) would find a challenge in multi-morphemic ISPs. Since simple ISPs seem to appear in higher or lower positions, assigning a singular structural analysis would be complicated, perhaps involving ad hoc stipulations about movement. Thus, examples (8)-(18) and (37)-(40) remain unaccounted for. Second, these proposals do not address the differences in the distribution of simple ISPs, such as $a$ versus $d i$, as discussed via (19)-(26), (32)-(36) and (41)-(43). Inversion data also suggest that the distribution of these ISPs is context-sensitive, since the projective ISPs fronte and destra must occur with $d i$ and a (e.g., di fronte), lest the syntactic string be ungrammatical.

Third, neither of the proposals tackles the locative inversion and argument demotion data in detail, nor how these phenomena are licensed. Cartographic proposals may offer a partial solution, as they offer simple and principled accounts on how argument demotion can occur (cf. Svenonius 2008; 2010), but locative inversion data involving complex ISPs would provide yet another challenge. Fourth, neither of these proposals lends itself easily to a fully compositional semantic analysis. Both Tortora (2008) and Folli (2008) start from problematic assumptions on aspect, but cartographic-driven analysis such as Svenonius (2008) would run into similar problems once it is extended to our full data set of ISPs. We are still in need of a fully compositional analysis of ISPs. In the next two sections, then, we offer a solution to these problems.

\section{The proposal: Morphological assumptions and analyses}

The goal of this section is to present the apparatus that we employ to offer a solution to our four morphological problems. We first introduce DM as our model of grammar, and offer a Type-Logical Syntax formalization, which builds on previous similar work (Ursini 2011; 2013; 2015; Ursini \& Akagi 2013a; section 3.1). We then discuss how we apply it to the data, and how it solves our first three problems (section 3.2). We conclude by discussing how the analysis improves over previous models (section 3.3). 


\subsection{The proposal: Morphological assumptions and Type-Logical DM}

In order to account our morphosyntactic problems, we adopt a combination of two seemingly unrelated frameworks: DM and Type-Logical Syntax (henceforth TLS). We choose DM as our model of grammar, since it postulates an explicit relation between morphosyntactic derivations, semantic interpretation and phonological insertion (Embick \& Noyer 2006; Harbour 2007; Harley 2010a;b; 2012). DM also takes a flexible approach to morphosyntactic categories, since it assumesthat functional elements can instantiate different structural templates. Since only few DM works analyse (English) SPs (Thomas 2001; 2003; 2004), our analysis also attempts to extend this account to other SP data. Three key assumptions play a role, in DM.

First, morphology and syntax form a single derivational system that governs word-, phrase- and sentence-formation. Thus, the same principles derive the structure of the ISP alla 'at-the' and the phrase di fronte alla finestra 'in front of the window'. Second, morphemes correspond to clusters/sets of features. In turn, these clusters can correspond to different morphosyntactic categories. Two factors play a key role: the first is the combination of features that a morpheme realises; the second is valence, the "amount" of arguments that a morpheme, qua a head, can take. While the definite article $l a$ 'the' amounts to the clustering of number, gender (female) and definiteness features, $a$ 'at/to' includes "spatial" features, and acts as a 1-place predicate (valence). Third, once morphological objects are derived, the output is mapped onto both the semantic and phonological components of grammar. Hence, the senses of sentences are computed via simple operations, while phonological rules determine the insertion and "combination" of vocabulary items/exponents (e.g., RS).

In order to formally represent these assumptions, in particular the notion of "derivation", we use core aspects of TLS and its treatment of morphosyntactic operations (Moortgat 2010; 2011; Morrill 2011). We start by defining the basic building block used in our derivations. In TLS, morphosyntactic categories are mapped or assigned onto types, which are represented as being either "complete" or "incomplete" information units. Complete types represent derivational elements that can stand as distinct, independent elements, e.g., $n p$ for noun phrases as the girl. Incomplete types are elements that must combine with other elements to form a complete type, e.g., $n p / s$ for the intransitive verb runs. When the girl combines with runs to form the sentence the girl runs, its type is $s$, as the two types $n p$ are "cancelled out". We use the convention of calling $n p$ and $s$, in the complex type $n p / s$, the input and the output types, respectively. 
We use the connectives "/" and "•" to represent the right division (or "slash") and the product operations (Moortgat 2010, §2; Morrill 2011: ch. 1). We define right division as a binary, associative operation, and product as a non-commutative operation: $\boldsymbol{a} \bullet \boldsymbol{b}$ is made of the ordered pair $\boldsymbol{a}$ and $\boldsymbol{b}$. We only implement the right-associative version of division, "/", and assume that derivations compute information about types in a top-down manner. For instance, di fronte 'in front' is merged with alla 'of the' before scrivania 'table' in (40). This assumption is consistent with psychological models of word production (Levelt 1989; Phillips 2006; Jarema \& Libben 2007), and will allow us to account RS and other derivational phenomena, as we show in in section 3.2. Thus, while (right) division can define the order of types assigned to lexical items, product defines whether each unit in a derivation includes combined (bundled) types. We leave aside the possibility that other connectives can be implemented (e.g., the standard left division/slash "Y", the connective "" for anaphors: Jäger 2005).

We then make a novel assumption on the basic set of atomic types in our lexicon, based on current findings and analyses of lexical categories in DM. These analyses suggest that traditional categories emerge as the result of merging categoryless roots with category-assigning elements, such as little $v, p$, and $n$ (Harbour 2007; Harley 2010a; 2012). We drop naïve types such as $s, n p$, and assume only one atomic type $\boldsymbol{p}$, mnemonic for "phrase", from which other types are recursively defined. We can now define our set of types via the application rules in (50):

(50) 1. Given a Lexicon L, $\boldsymbol{p}$ is a morphological type

(Lexical type)

2. If $\boldsymbol{x}$ is a type and $\boldsymbol{y}$ is a type, then $\boldsymbol{x} / \boldsymbol{y}$ is a type

(Type formation: division)

3. If $\boldsymbol{x}$ is a type and $\boldsymbol{y}$ is a type, then $\boldsymbol{x} \bullet \boldsymbol{y}$ is a type

(Type formation: product)

4. If $\boldsymbol{x} / \boldsymbol{y}$ is a type and $\boldsymbol{y}$ is a type, then $(\boldsymbol{x} / \boldsymbol{y}) \cdot \boldsymbol{y} \vdash \boldsymbol{x} ; \boldsymbol{y} \bullet(\boldsymbol{x} / \boldsymbol{y}) \vdash \boldsymbol{x}$ (Merge: forward a.)

5. If $\boldsymbol{x} / \boldsymbol{y}$ is a type and $\boldsymbol{y} / \boldsymbol{z}$ is a type, then $(\boldsymbol{x} / \boldsymbol{y}) \bullet(\boldsymbol{y} / \boldsymbol{z}) \vdash \boldsymbol{x} / \boldsymbol{z}$

(Merge: cut rule)

6. Nothing else is a type

(Closure rule)

Rule 1 defines our basic type $\boldsymbol{p}$ as the key building block on which more complex types are built, via the two operations (right) division and product. Rules 2 and 3 define how more complex types representing heads or complex morphemes are formed, respectively. Rule 2 allows for the definition of the so-called "functional" types, which merge with other types to yield an output type: for instance, la 'the' merges with the NP casa 'house' to form the DP la casa 'the house'. Rule 3 allows for the definition of product types and entails that basic features are assigned the type $\boldsymbol{p}$ 
of phrases, which perhaps can also be seen as mnemonic for predicates in this case.

Rules 4 and 5 define how morphemes interact to form more complex structures (e.g., phrases, conflated heads). Rule 4 is based on the principle of forward application (Moortgat 2011, §2.1; Morryll 2011, ch. 1): adjacent and matching types are "cancelled out". If two types do not match (e.g., we merge $\boldsymbol{x}$ and $\boldsymbol{y}$ ), a derivation is said to diverge or crash. Thus, the connective "-" says that, if we take two inputs of a certain type, then we can prove that their result is an output of a certain type. Rule 5 is the cut rule (also known as the transitivity rule, Moortgat 2011, §2.1), a rule that allows for the combination of types with the same valence, at least in our basic definition. Anticipating matters a bit, while rule 4 governs the merge of items such as alla and casa to for the ISP phrase alla casa 'at the house', rule 5 governs the merge of $a$ and la into alla. Rule 6 (the closure rule) says that no rules can derive types, in our system.

Rules 4-5 represent TLS counterparts of the merge operation in DM. TLS and minimalism share the common assumption that merge is a ternary relation $R x y z$ between two inputs $x$ and $y$ (head and argument) and output $z$ (the resulting phrase). In our TLS system, the merge operation operates in a "distributed" manner: the product of possibly complex types proves the existence of a larger constituent. Since different constituents can be combined via product, if their types match, different instances of merge can be defined (forward application, cut rule). This is consistent with DM (Harbour 2007, ch. 1-2), but also with other minimalist frameworks (e.g., Hale \& Keyser 2002).

One further rule that will play a role in our analysis is the residuation rule. The residuation rule is defined as: if $\boldsymbol{x} \bullet \boldsymbol{y}$ is a type and $\boldsymbol{z}$ is a type, then $(\boldsymbol{x} \bullet \boldsymbol{y}) / \boldsymbol{z} \vdash \boldsymbol{x} / \boldsymbol{y} / \boldsymbol{z}$. In words, a type $y$ of a product type $\boldsymbol{x} \bullet \boldsymbol{y}$ can become an input type, hence becoming able to merge with other (input) types. This rule captures valence differences that functional heads can have in different syntactic contexts; hence, it plays a role in our analysis of $d i$ 'of' and other simple ISPs in their affix-like distribution (e.g., di fronte 'in front'). Via this set of assumptions, we can generate the minimal type set $\boldsymbol{T Y P E}=$ $\{\boldsymbol{p}, \boldsymbol{p} / \boldsymbol{p}, \boldsymbol{p} / \boldsymbol{p} / \boldsymbol{p}, \boldsymbol{p} \bullet \boldsymbol{p}, \ldots\}$, with $\boldsymbol{p} / \boldsymbol{p} / \boldsymbol{p}$ being short for $(\boldsymbol{p} /(\boldsymbol{p} / \boldsymbol{p}))$. While $\boldsymbol{p}$ is the type of phrases/arguments (e.g., the car), $\boldsymbol{p} / \boldsymbol{p} / \boldsymbol{p}$ is the type of heads qua relational elements (e.g., of), and the type $\boldsymbol{p} / \boldsymbol{p}$ is that of affixlike morphemes. The product type $\boldsymbol{p} \bullet \boldsymbol{p}$ can involve $n$ basic types, but for the sake of simplicity we only use this basic binary type.

In order to capture the cyclical nature of our derivations, we define a simple pre-order as the pair of an interval set $I$, and an addition operation 
"+", i.e., $\langle I,+\rangle$. This pre-order represents an index set, which allows for representations of the steps in a derivation as ordered elements. We implement two operations, lexical selection (LS) and merge introduction (MI), to explicitly mark the introduction of a new item in a derivation and the merging of two items, respectively. A sample derivation is in (51):

(51) a. Mario loves Peach.

$$
\begin{aligned}
& \text { b. t. }\left[\operatorname{Mario}_{\boldsymbol{p}}\right] \\
& \left.t+1 \text {. [ } \text { loves }_{\boldsymbol{p} / \boldsymbol{p} / \boldsymbol{p}}\right] \\
& t+2 \text {. }\left[\text { Mario }_{\boldsymbol{p}}\right] \bullet\left[\operatorname{loves}_{\boldsymbol{p} / \boldsymbol{p} / \boldsymbol{p}}\right] \vdash\left[\boldsymbol{p} / \boldsymbol{p}\left[\text { Mario }_{\boldsymbol{p}}\right] \operatorname{loves}_{\boldsymbol{p} / \boldsymbol{p} / \boldsymbol{p}}\right] \\
& t+3 \text {. }\left[\text { Peach }_{\boldsymbol{p}}\right] \\
& t+4 .\left[{ }_{\boldsymbol{p} / \boldsymbol{p}}\left[\operatorname{Mario}_{\boldsymbol{p}}\right] \operatorname{loves}_{\boldsymbol{p} / \boldsymbol{p} / \boldsymbol{p}}\right] \bullet\left[\text { Peach }_{\boldsymbol{p}}\right] \vdash\left[{ }_{\boldsymbol{p}}\left[\text { Mario }_{\boldsymbol{p}}\right] \operatorname{loves}_{\boldsymbol{p} / \boldsymbol{p} / \boldsymbol{p}}\left[\operatorname{Peach}_{\boldsymbol{p}}\right]\right]
\end{aligned}
$$

This simplified derivation reads as follows. A phrasal element, the DP Mario, is merged with the transitive verb loves. Since the first element is assigned type $\boldsymbol{p}$ and the second type $\boldsymbol{p} / \boldsymbol{p} / \boldsymbol{p}$, the merge of these elements is assigned type $\boldsymbol{p} / \boldsymbol{p}$, as a result of this derivational process. The further merge of Peach allows the full sentence Mario loves Peach to be formed, an "skeletal" VP of type $\boldsymbol{p}$. Leaving functional projections aside, this can be seen as the type of VP that the two constituents form via a merge. Once we have defined our formal apparatus in detail, we can focus on our analysis of the data.

\subsection{The analysis: An analysis of the morphology of ISPS}

The goal of this section is to explain how our analysis can account for the data at hand, hence offering a solution to our three morphosyntactic problems. We start by discussing which structural analysis we assign to ISPs, and from complex ISPs, for practical reasons. Recall from (10)-(11) that complex ISPs can undergo argument demotion; hence, projective ISPs can occur as arguments of verbs. Consider (43), repeated as (52):

(52) Dietro (alla/la stazione), Mario fuma una sigaretta.

behind (at-the/the station) Mario smokes a cigarette

As the example shows, the projective ISP dietro can involve demotion, and can occur in an inverted position; it seems to act as an argument/phrase, in this distinct syntactic conctext. Therefore, dietro and other projective ISPS are assigned type $\boldsymbol{p}$ of phrases. In the case of a multi-morphemic ISP such as di fronte 'in front' (viz. (41)), the ISP's ability to also occur in an 
inverted position suggests that its type is $\boldsymbol{p}$. Since fronte as a projective ISP must be assigned type $\boldsymbol{p}$, an obvious but preliminary conclusion is that $d i$ and similarly $a$ are of type $\boldsymbol{p} / \boldsymbol{p}$ : the merge of the two elements would give the type $\boldsymbol{p}$ as a result. The case of $a$ in the simple ISP alla 'at-the' will require some more clarification.

The next problem we discuss to clarify this point, then, is the type assignment for the other morphemes making up a complex ISP. We focus on del 'of-the', which allows us to offer our treatment of RS. For the definite articles $i l$ and $-l$, we take their type to be $\boldsymbol{p \bullet p / p}$ (cf. Ritter 1991; 1993; Harbour 2007, ch. 3), a 1-place predicate. We take that determiners are the locus of definiteness and merge with a ground DP, an object of type $\boldsymbol{p}$ in our assignment, to form a phrase marked for definiteness. This feature value is represented via the type $\boldsymbol{p} \bullet \boldsymbol{p}$, which is the output type of a DP such as la finestra 'window', which also carries gender and number features, as a product type.

We move to our relational elements, including $d i$ and $a$ as heads. A standard assumption about $d i$ 'of' is that it acts as a prototypical relational head, hence it can be assigned the type $\boldsymbol{p} \bullet \boldsymbol{p} / \boldsymbol{p} / \boldsymbol{p} \bullet \boldsymbol{p}$ (a "nominal" copula, den Dikken 2006, ch. 4-5). We take a similar stance on $a$,). Hence assuming that both ISPs can be polymorphic: their type assignment depends on the syntactic context they occur in (Morrill 2011, ch. 2; Moortgat $2011, \S 3)$. The residuation rule tells us that this polymorphism is highly constrained, since a relational morpheme (type $\boldsymbol{p} / \boldsymbol{p} / \boldsymbol{p}$ ) can only derived from an affix-like morpheme (type $\boldsymbol{p} \boldsymbol{p} / \boldsymbol{p}$ ). Hence, the apparently different types for $a$ are tightly related, and the type for $a$ as an affix is now justified, and will soon be shown to make precise predictions about our data. We can now offer our type assignment in (53):

(53) a. $\boldsymbol{p} \bullet \boldsymbol{p} / \boldsymbol{p} / \boldsymbol{p} \bullet \boldsymbol{p}::=\left\{d i, d e-, d e l, d a,(P),(P)^{\prime}\right.$, al, alla, $\left.\ldots\right\}$

b. $\boldsymbol{p}::=\{$ scrivania, tavolo, fronte, dietro, di fronte alla finestra, ... $\}$

c. $\boldsymbol{p} \bullet \boldsymbol{p} / \boldsymbol{p}::=\{a, i l, l a, d i,-l, n e-, \ldots\}$

d. $\boldsymbol{p} \bullet \boldsymbol{p}::=\{$ lui, davanti, dietro, sopra, di fronte, $(p), \ldots\}$

In words, the type $\boldsymbol{p} \bullet \boldsymbol{p} / \boldsymbol{p} / \boldsymbol{p} \bullet \boldsymbol{p}$ is defined as the type of simple ISPs, in their relational distribution. Thus, it includes $a$ and $d i$, but also $d a$ 'from', alla and the silent ISP "(P)". The product input types reflect that ISPs cannot combine with just any constituents, but only with those that carry "spatial" features. Hence, while davanti a 'ahead of' is a possible (complex) ISP, * giallo a 'yellow at' is not. The type $\boldsymbol{p}$ is the type of DPs such as scrivania 'desk', but also of "bare" projective/Axpart ISPs such as fronte, and of full phrases such as di fronte alla finestra 'in front of the window'. 
The type $\boldsymbol{p} \boldsymbol{\bullet} / \boldsymbol{p}$ is the type of affix-like functional elements, such as $a$ and the definite article. These items can combine with a nominal element (scrivania 'table' and fronte 'front', for instance), to form phrases carrying "spatial" and "definite" features, respectively (e.g., di fronte, la scrivania). The type $\boldsymbol{p} \bullet \boldsymbol{p}$ is reserved for phrases such as di fronte 'in front' or the pronoun lui 'him', for reasons that will soon become clear. We now repeat (37) as (54a):

(54) a. Mario siede di fronte alla scrivania. 'Mario sits in front of the desk.'

b. $t . \quad\left[\mathrm{di}_{\boldsymbol{p} \bullet \boldsymbol{p} / \boldsymbol{p}}\right]$

$t+1$. $\left[\right.$ fronte $\left._{\boldsymbol{p}}\right]$

$t+2 .\left[\mathrm{di}_{\boldsymbol{p} \bullet \boldsymbol{p} / \boldsymbol{p}}\right] \bullet\left[\right.$ fronte $\left._{\boldsymbol{p}}\right] \vdash\left[\boldsymbol{p} \bullet \boldsymbol{p}\right.$ di $\mathrm{p}_{\boldsymbol{p} \boldsymbol{p} / \boldsymbol{p}}\left[\right.$ fronte $\left.\left._{\boldsymbol{p}}\right]\right]$

$t+3 \cdot\left[\mathrm{a}_{\boldsymbol{p} \bullet \boldsymbol{p} / \boldsymbol{p} / \boldsymbol{p} \bullet \boldsymbol{p}}\right]$

$t+4 .\left[\boldsymbol{p} \bullet \boldsymbol{p}\right.$ di $\boldsymbol{p \bullet p / p}\left[\right.$ fronte $\left.\left._{\boldsymbol{p}}\right]\right] \bullet\left[\mathrm{a}_{\boldsymbol{p} \bullet \boldsymbol{p} / \boldsymbol{p} / \boldsymbol{p} \bullet \boldsymbol{p}}\right] \vdash$

$\left[\boldsymbol{p} / \boldsymbol{p} \bullet \boldsymbol{p}\left[\boldsymbol{p \bullet p} \mathrm{di}_{\boldsymbol{p} \bullet \boldsymbol{p} / \boldsymbol{p}}\left[\right.\right.\right.$ fronte $\left.\left._{\boldsymbol{p}}\right]\right]$ a $\left.\boldsymbol{p \bullet p / p / p \bullet p}\right]$

$t+5 .\left[\mathrm{la}_{\boldsymbol{p} \bullet \boldsymbol{p} / \boldsymbol{p}}\right]$

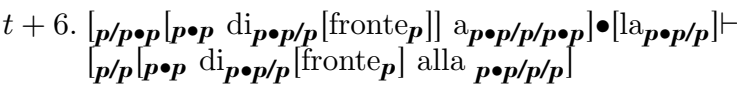

$t+7$. [scrivania $p]$

(MI: Cut Rule)

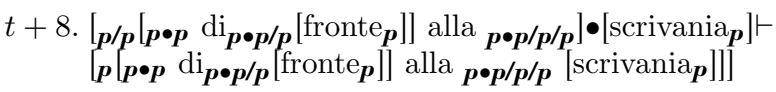

In words, in (54b) the two morphemes di (here, 'in') and fronte 'front' merge first, forming a phrase (di fronte 'in front') that is then merged with $a$ (here, 'of'). Since $d i$ is assigned type $\boldsymbol{p} \bullet \boldsymbol{p} / \boldsymbol{p}$ via the residuation rule, the di fronte is of type $\boldsymbol{p} \bullet \boldsymbol{p}$. Since we also restrict the argument types for $a$ to product types, we predict that di fronte 'in front', but not fronte 'front', can merge with $a$. By step $t+4$, we have an object of type $\boldsymbol{p} / \boldsymbol{p} \bullet \boldsymbol{p}$, di fronte $a$ 'in front of'. The determiner la merges next, via the cut rule. Since di fronte $a$ and $l a$ have the same "internal" type (i.e., $\boldsymbol{p} \bullet \boldsymbol{p}$ ), they can only combine by "eliminating" structure, forming one morphological object. Thus, we suggest that RS is a phonological reflex of this morphological process, by which the cut structure is "recorded" via the conflated exponents: we have di fronte alla 'in front of-the'. The derivation ends when the DP scrivania 'desk' of type $\boldsymbol{p}$ is merged, forming the ISP di fronte alla scrivania.

Via this result, we obtain at least two other results. First, we can show that product types can be used to explain selectional restrictions on the argument of a head, but without assuming an independent argument structure and "c-selection" principles (cf. Harley 2010b). While "bare" fronte 'front' cannot merge with alla 'of-the', non-bare di fronte 'in front' carries 
the relevant features that permit this phrase to merge with its head. Second, we also straightforwardly capture feature-matching analyses found in the literature, via the basic principle of type matching that merge requires (Harbour 2007, ch. 2-3; Adger 2010). However, since our data only involve simple cases of restrictions, we can avoid introducing index systems for product types (e.g., $\left.\boldsymbol{p} \bullet \boldsymbol{p}_{\text {pers }}\right) .^{8}$

Furthermore, we also show that our top-down approach avoids one complication that would emerge in a bottom-up approach. That is, fronte would be merged before $d i$, and an ungrammatical string would be derived (*fronte alla scrivania) before the grammatical di fronte 'in front' would be derived. In our approach, this problem never arises, since di fronte is derived before the rest of the ISP phrase. One more datum in our support is that the structure of multi-moprhemic ISPs can receive an identical analysis. We repeat (39) as (55a) to discuss this datum:

(55) a. Mario siede nel mezzo della stanza.

'Mario sits in the middle of the room.'

b. $t . \quad\left[\mathrm{ne}_{\boldsymbol{p} \bullet \boldsymbol{p} / \boldsymbol{p}}\right]$

$t+1 .\left[\mathrm{il}_{\boldsymbol{p} \bullet \boldsymbol{p} / \boldsymbol{p}}\right]$

$t+2 . \quad\left[\mathrm{ne}_{\boldsymbol{p} \bullet \boldsymbol{p} / \boldsymbol{p}}\right] \bullet\left[\mathrm{il}_{\boldsymbol{p} \bullet \boldsymbol{p} / \boldsymbol{p}}\right] \vdash\left[\mathrm{nel}_{\boldsymbol{p} \bullet \boldsymbol{p} / \boldsymbol{p}}\right]$

$t+3 . \quad\left[\operatorname{mezzo}_{\boldsymbol{p}}\right]$

$t+4 . \quad\left[\operatorname{nel}_{\boldsymbol{p} \bullet \boldsymbol{p} / \boldsymbol{p}}\right] \bullet\left[\operatorname{mezzo}_{\boldsymbol{p}}\right] \vdash\left[\boldsymbol{p} \bullet \boldsymbol{p}\left[\operatorname{nel}_{\boldsymbol{p} \bullet \boldsymbol{p} / \boldsymbol{p}}\left[\operatorname{mezzo}_{\boldsymbol{p}}\right]\right]\right.$

$t+5$. $\left[\mathrm{de}_{\boldsymbol{p} \bullet \boldsymbol{p} / \boldsymbol{p} / \boldsymbol{p} \bullet \boldsymbol{p}}\right]$

$t+6 . \quad\left[\boldsymbol{p} \bullet \boldsymbol{p}\left[\operatorname{nel}_{\boldsymbol{p} \bullet \boldsymbol{p} / \boldsymbol{p}}\left[\operatorname{mezzo}_{\boldsymbol{p}}\right]\right] \bullet\left[\mathrm{de}_{\boldsymbol{p} \bullet \boldsymbol{p} / \boldsymbol{p} / \boldsymbol{p} \bullet \boldsymbol{p}}\right] \vdash\right.$

$t+7$. $\left[-11 \mathrm{a}_{\boldsymbol{p} \bullet \boldsymbol{p} / \boldsymbol{p}]}\right]$

$t+8 . \quad\left[\boldsymbol{p} / \boldsymbol{p} \bullet \boldsymbol{p}\left[\boldsymbol{p} \bullet \boldsymbol{p}\left[\mathrm{nel}_{\boldsymbol{p} \bullet \boldsymbol{p} / \boldsymbol{p}}\left[\mathrm{mezzo}_{\boldsymbol{p}}\right]\right] \mathrm{de}_{\boldsymbol{p} \bullet \boldsymbol{p} / \boldsymbol{p} / \boldsymbol{p} \bullet \boldsymbol{p}}\right]\right] \bullet\left[-1 \mathrm{la} \mathrm{p}_{\boldsymbol{p} \boldsymbol{p} / \boldsymbol{p}}\right] \vdash$

$t+9 . \quad\left[\operatorname{stanza}_{\boldsymbol{p}}\right]$

$t+10 .\left[\boldsymbol{p} / \boldsymbol{p} \bullet \boldsymbol{p}\left[\boldsymbol{p} \bullet \boldsymbol{p}\left[\operatorname{nel}_{\boldsymbol{p} \bullet \boldsymbol{p} / \boldsymbol{p}}\left[\operatorname{mezzo}_{\boldsymbol{p}}\right]\right]\right.\right.$ della $\left.\left.\boldsymbol{p \bullet p / p / p}\right]\right] \bullet\left[\operatorname{stanza}_{\boldsymbol{p}}\right] \vdash$ $\left[\boldsymbol{p}\left[\right.\right.$ nel $_{\boldsymbol{p} \bullet \boldsymbol{p} / \boldsymbol{p}}\left[\right.$ mezzo $\left.\left._{\boldsymbol{p}}\right]\right]$ della $\boldsymbol{p} \bullet \boldsymbol{p} / \boldsymbol{p} / \boldsymbol{p}\left[\right.$ stanza $\left.\left._{\boldsymbol{p}}\right]\right]$

The derivation shows that, with our type assignment and use of merge, we can predict that the two morphemes ne and $i l$ undergo RS, since they can be merged via the cut rule. Thus, our treatment of RS contrasts with that with Napoli \& Nevis (1987), who treat conflated ISPs as "monadic" lexical

${ }^{8}$ In Adger (2010, 190-196), feature matching involves two equivalent features (and their values) $\alpha$ and $\beta$ : we have $\alpha=\beta$. In TLS, matching is part of the definition of merge. However, see TLS works such as Johnson \& Bayer 1995, for flexible treatments of feature-matching processes. 
items, hence distinct from their simple ISP counterparts. Our approach is, on the other hand, consistent with standard DM assumptions about "post-syntactic" operations (Embick \& Noyer 2001; Harbour 2007). The phonological realization of in 'in' and $l a$ 'the' as the allomorph nella 'in-the' is a consequence of the merge of the two morphemes apparently creating an exponent that is unattested in Italian (i.e., ${ }^{*}$ inla). Thus, nella is inserted after merge creates a new object.

Further support comes from speech production data in healthy and aphasic patients (Franco \& Zampieri 2012). Aphasic patients usually omit the definite article but not the simple ISP $a$, when elicited to produce complex ISPs; healthy participants may also do so, when production mistakes crop up. Both types of findings suggest that conflated ISPs are indeed the result of merging two basic exponents, which then undergo RS. Hence, our analysis seems to offer an account of RS that is also consistent with experimental findings, and can now account examples (8)-(18) and (37)-(40) and their respective classes. Before we offer further considerations on this fact, we show how we can analyse the other data. We repeat (13) and (8) as (56a)-(57a) to illustrate this result:

(56) a. Mario è davanti al tavolo da biliardo. 'Mario is in front of the pool table.'

b. $t . \quad[$ davanti $p \bullet p]$

$t+1 . \quad\left[\mathrm{al}_{\boldsymbol{p} \bullet \boldsymbol{p} / \boldsymbol{p} / \boldsymbol{p}}\right]$

$t+2 . \quad\left[\right.$ davanti $\left._{\boldsymbol{p} \bullet \boldsymbol{p}}\right] \bullet\left[\mathrm{al}_{\boldsymbol{p} \bullet \boldsymbol{p} / \boldsymbol{p} / \boldsymbol{p}}\right] \vdash\left[\boldsymbol{p} / \boldsymbol{p}\left[\operatorname{davanti}_{\boldsymbol{p} \bullet \boldsymbol{p}}\right] \mathrm{al}_{\boldsymbol{p} \bullet \boldsymbol{p} / \boldsymbol{p} / \boldsymbol{p}}\right]$

$t+3$. [ tavolo $\left._{\boldsymbol{p}}\right]$

$t+4 . \quad\left[\boldsymbol{p} / \boldsymbol{p}\left[\right.\right.$ davanti $\left._{\boldsymbol{p} \bullet \boldsymbol{p}}\right]$ al $\left._{\boldsymbol{p} \bullet \boldsymbol{p} / \boldsymbol{p}}\right] \bullet\left[\right.$ tavolo $\left._{\boldsymbol{p}}\right] \vdash\left[\boldsymbol{p}\left[\right.\right.$ davanti $\left._{\boldsymbol{p} \bullet \boldsymbol{p}}\right]$ al $\left._{\boldsymbol{p} \bullet \boldsymbol{p} / \boldsymbol{p}}\left[\operatorname{tavolo}_{\boldsymbol{p}}\right]\right]$

(57) a. Mario è al tavolo da biliardo. 'Mario is at the pool table.'

b. $t . \quad\left[(\mathrm{p})_{\boldsymbol{p} \bullet \boldsymbol{p}}\right]$

$t+1 . \quad\left[\mathrm{al}_{\boldsymbol{p} \bullet \boldsymbol{p} / \boldsymbol{p} / \boldsymbol{p}}\right]$

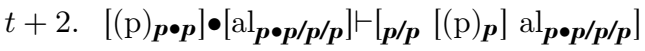

$t+3$. [tavolop $]$

$t+4 . \quad\left[\boldsymbol{p} / \boldsymbol{p}[(\boldsymbol{p}) \boldsymbol{p}] \mathrm{al}_{\boldsymbol{p} \bullet \boldsymbol{p} / \boldsymbol{p} / \boldsymbol{p}}\right] \bullet\left[\operatorname{tavolo}_{\boldsymbol{p}}\right] \vdash\left[\boldsymbol{p}\left[(\mathrm{p})_{\boldsymbol{p}}\right] \mathrm{al}_{\boldsymbol{p} \bullet \boldsymbol{p} / \boldsymbol{p} / \boldsymbol{p}}\left[\right.\right.$ tavolo $\left.\left._{\boldsymbol{p}}\right]\right]$

As the derivations show, the minimal differences between these ISPs and the complex ISPs are that only one morpheme, a projective ISP, is merged first. Note that complex ISPs seem to mostly comprise "compound" projective ISPs. This suggests that they correspond to the type $\boldsymbol{p} \bullet \boldsymbol{p}$. Since projective morphemes (davanti) receive the same type, $\boldsymbol{p} \boldsymbol{p}$, as their slightly 
more complex counterparts (di fronte). ${ }^{9}$ In the case of al in (57), we assume that a silent projective morpheme "(p)" is merged with al.

With these results at our disposal, we can now tackle examples (19)(23), which involve pronouns as ground DPs (lui 'him'), and which in turn can only merge with $d i$ and not with $a$. In our analysis, a natural explanation is that both pronouns and ISPs carry the same value for the input product type, so they can merge successfully. Since a silent "(P)" does not carry the same value, it cannot merge with pronouns, lest the derivation crashes. We repeat (19) as (58a) and offer the derivations in $(58 \mathrm{~b}-\mathrm{c})$ :

(58) a. Mario è dietro di $/ *(\mathrm{P})$ lui.

'Mario is behind of $/ *(\mathrm{P})$ him.'

b. $t$. [dietropøp]

$t+1 . \quad\left[\mathrm{di}_{\boldsymbol{p} \bullet \boldsymbol{p} / \boldsymbol{p} / \boldsymbol{p} \bullet \boldsymbol{p}}\right]$

$t+2 . \quad\left[\operatorname{dietro}_{\boldsymbol{p} \bullet \boldsymbol{p}}\right] \bullet\left[\operatorname{di}_{\boldsymbol{p} \bullet \boldsymbol{p} / \boldsymbol{p} / \boldsymbol{p} \bullet \boldsymbol{p}}\right] \vdash\left[\boldsymbol{p} / \boldsymbol{p} \bullet \boldsymbol{p}\left[\operatorname{dietro}_{\boldsymbol{p} \bullet \boldsymbol{p}}\right] \operatorname{di} \boldsymbol{p} \boldsymbol{p} / \boldsymbol{p} / \boldsymbol{p} \bullet \boldsymbol{p}\right]$

$t+3$. [lui $p \bullet p]$

$t+4 . \quad\left[\boldsymbol{p} / \boldsymbol{p} \bullet \boldsymbol{p}\left[\operatorname{dietro}_{\boldsymbol{p} \bullet \boldsymbol{p}}\right] \mathrm{di}_{\boldsymbol{p} \bullet \boldsymbol{p} / \boldsymbol{p} / \boldsymbol{p} \bullet \boldsymbol{p}}\right] \bullet\left[\operatorname{lui}_{\boldsymbol{p} \bullet \boldsymbol{p}}\right] \vdash$

c. $\left.t+4 . \quad{ }_{\boldsymbol{p} / \boldsymbol{p} \bullet \boldsymbol{p}}\left[\operatorname{dietro}_{\boldsymbol{p} \bullet \boldsymbol{p}}\right](\mathrm{P})_{\boldsymbol{p} / \boldsymbol{p} / \boldsymbol{p} \bullet \boldsymbol{p}}\right] \bullet\left[\operatorname{lui}_{\boldsymbol{p} \bullet \boldsymbol{p}}\right]^{* *}$

(Derivation crashes)

The derivation in (58) shows what would happen if lui 'him' would merge with an ISP lacking the correct type. In order to see why this is the case, suppose that "(P)" also includes a compound type, which we however call "p•p". If lui merges with "(P)", then, a feature mismatch occurs, as we have "p•p" to merge with $\boldsymbol{p} \boldsymbol{p}$. The derivation cannot continue: a string is correctly predicted to be ungrammatical, as shown in the partial derivation (58c). A similar approach can be extended to $a$ in inverted/extracted positions (i.e., (24)-(26)), to the distribution of $d a$ with animate ground DPs in (32)-(36), and additionally to coordination data in (22)-(23). Since $e$ 'and' and $o$ 'or' are syn-cagorematic elements, they must have the type $\boldsymbol{p}$ of ISP phrases on both input types. Hence, while di me 'of me' and $d i$ lui 'of him' can act as arguments of conjunctions and disjunctions, bare pronouns cannot occur, as per predictions.

Since we now have derived full ISP phrases, we can show how these phrases can occur in an inverse position, and how arguments can be demoted. We repeat (41) as (59a) to show our (compressed) analysis of these inversion cases:

${ }^{9}$ A caveat: the actual type for complex ISPs should be more complex, and obtained via two applications of the residuation rule (i.e., we have $\boldsymbol{p} \bullet \boldsymbol{p} \bullet \boldsymbol{p})$. Idempotence licenses a simpler notation (i.e., $\boldsymbol{p} \bullet \boldsymbol{p} \bullet \boldsymbol{p} \vdash \boldsymbol{p} \bullet \boldsymbol{p}$ ). 
(59) a. Di fronte alla finestra, I ragazzi bevono birra.

'In front of the window, the boys drink beer.'

b. $t . \quad[\boldsymbol{p}$ di fronte alla... $]$

$t+1 \cdot\left[\left(\mathrm{P}^{\prime}\right)_{\boldsymbol{p} / \boldsymbol{p} / \boldsymbol{p}}\right]$

$t+2 . \quad[\boldsymbol{p}$ di fronte alla... $] \bullet\left[\left(\mathrm{P}^{\prime}\right)_{\boldsymbol{p} / \boldsymbol{p} / \boldsymbol{p}}\right] \vdash\left[\boldsymbol{p} / \boldsymbol{p}[\right.$ pdi fronte alla... $\left.]\left(\mathrm{P}^{\prime}\right)_{\boldsymbol{p} / \boldsymbol{p} / \boldsymbol{p}}\right]$

$t+3$. [I ragazzi...p]

$t+4 .\left.\quad\left[\boldsymbol{p} / \boldsymbol{p}[\right.$ di fronte alla...p $\left.]\left(\mathrm{P}^{\prime}\right)_{\boldsymbol{p} / \boldsymbol{p} / \boldsymbol{p}}\right] \bullet\right|_{\boldsymbol{p}}$ i ragazzi... $] \vdash$

$\left[\boldsymbol{p}[\right.$ di fronte alla finestra $\boldsymbol{p}]\left(\mathrm{P}^{\prime}\right)_{\boldsymbol{p} / \boldsymbol{p} / \boldsymbol{p}}[\boldsymbol{p}$ I ragazzi...]]

In words, the fronted ISP phrase di fronte alla finestra, of type $\boldsymbol{p}$, is basegenerated and merged to the left of the silent head " $\left(\mathrm{P}^{\prime}\right)$ ", of type $\boldsymbol{p} / \boldsymbol{p} / \boldsymbol{p}$. We can avoid implementing any movement operations, which is in line with a top-down approach. Note that we also predict that also other types of ground DPs (e.g., a Luigi 'to Luigi' in (25)) can be in inverted position. The restriction on $a$ as being the only simple ISP that can occur in an inverted position, then, receives a similar structural analysis as that found in (58). Thus, the specific distribution of $a$, $d i$ and $d a$ can be seen as a reflex of which subsets of features are realised in a derivational context. This is, again, as per DM assumptions (Embick \& Noyer 2001; 2006; Harley 2010a;b; cf. also Adger 2010).

Another fact that finds a straightforward analysis is argument demotion. We take a standard account of argument demotion as a form of ellipsis (cf. Merchant 2001; 2004). Ellipsis targets an ISP phrase, and elides enough material to form another phrase, as seen in (10)-(11) and (42)-(43), Thus, in an ISP phrase such as di fronte alla finestra, la finesra can be elided, because the remaining morpheme di fronte 'in front' is phrasal in nature (i.e., type $\boldsymbol{p} \bullet \boldsymbol{p})$. Hence, our analysis predicts that di fronte, but not fronte 'front' can act as a "remnant" ISP. It also predicts that al cannot be involved in argument demotion as a stand-alone ISP, since it is of type $\boldsymbol{p} \boldsymbol{p} / \boldsymbol{p}$. Thus, our analysis can account for the examples in (23)-(31) via our TL approach. In fact, we now have an account of all our examples in (1)-(43), although their semantic account is still outstanding. Therefore, we have a solution to our first three problems, which are defined as follows.

Our first solution consists of a general theory on the structure of all of our classes of ISPs. Via our flexible approach to categories, it is possible to capture the distributional properties of dietro 'behind', davanti 'ahead' and other projective ISPs in a straightforward manner. Our second solution consists of a simple and systematic account of the distribution of simple ISPs $a, d a$ and $d i$. Our analysis can account for not only the ability of simple ISPs to appear in both "higher" and "lower" positions, but also 
simple ISPs' context-sensitive distribution. Under our account, the need for pronouns like lui 'him' to occur with the relational $d i$, rather than $a$, is a reflex of the different features that both items carry. If these features do not match, then the derivation crashes, as would be the case with $a$ or a silent P. hence, our third solution consists of a movement-free account for the locative inversion and argument demotion data, in turn based on the first and second solution.

\subsection{The morphological analysis: A discussion}

Let us take some stock, before we move to the semantic analysis. Our analysis of ISP heads and their distributional properties differs from previous analyses on a number of aspects. For instance, Axpart Ps such as dietro 'behind' and davanti 'ahead' are treated as phrases rather than heads, and are assigned to two different types: $\boldsymbol{p}$ and $\boldsymbol{p} \bullet \boldsymbol{p}$, respectively. Kase Ps such as $a$ and $d i$ are heads when occurring in a lower position, as in cartographic approaches (or the $\mathrm{R}$ head in Folli 2008; cf. (46b)). However, when these elements seem to project a Place head, they do so as affix-like elements, or more accurately as 1-place predicates.

Thus, it seems that our analysis is similar but not identical to the "P-within-P" analysis of Hale \& Keyser (2002, ch. 4), which suggests that SPs involve one relational $\mathrm{P}$ head, and a second and possibly complex ISP head sitting in its specifier position (cf. also Emonds 1985). However, our analysis is also close to that of Riemsdijk \& Huybregts (2007), which makes a similar but not identical proposal. In order to make these comparisons clear, we represent all these structures in (60). Two correspond to the ones that we can assign to the final output of (54). A third corresponds to Hale and Keyser's (2002) tentative approach; a fourth, to the structure we have worked with:

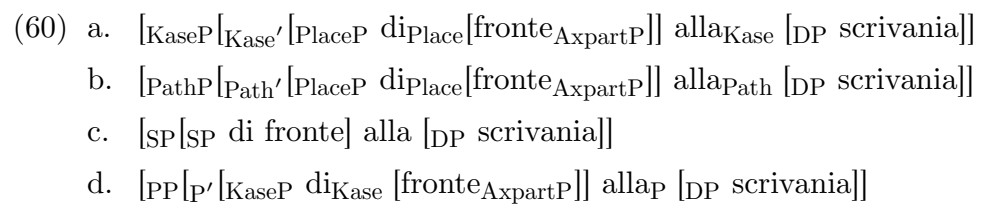

The structure in (60a) abstracts away from a Path head, since this head does not play a role in our analysis, and show that the categories Axpart, Place, and Kase have different valence. In particular, PlaceP sits in specifier position of KaseP, rather than taking this phrase as an argument. The structure in (60b) offers a view in which a Path head can denote inherently 
"static" relations. This structure is also reminiscent of proposals such as Emonds (1985) or Riemsdijk \& Huybregts (2007), which however do not include Axpart as part of SP structures. The structure in (60c) shows how a "P-within-P" analysis would apply to our example, complete with non-specific "SP" labels.

As our derivations show, ISPs involve at least two lexical items that can be considered as the principal units of their structure, as in the case of di fronte alla scrivania. The first unit, a phrase (i.e., di fronte 'in front'), can undergo fronting: this fact supports its status as a single unit. The same reasoning holds for alla scrivania 'at the desk', qua a unit that can be deleted, in argument demotion cases, but also fronted. Thus, our data suggest that the "P-within-P" hypothesis seems closer to our analysis. However, we have also observed that $d i$ and fronte must be merged as distinct lexical items, before they become the specifier phrase of alla. This fact brings our analysis closer to the structure in (60b), although one proviso is that our analysis of $d i$ ('in', in this case) treats this item as a Kase head. When $d i$ appears in this "position", it marks the nominal-like item it merges with as an element with a spatial preposition distribution and interpretation (i.e., Axpart fronte 'front' here).

The structure we envision for our ISPs, then, is the one proposed in (60d), in which a general or underspecified "P" head can either denote directional and locative relations. The underspecified nature of our ISPs in part justifies the lack of an explicit Place head. Thus, if we aim to maintain a certain stable mapping from syntactic categories to semantic interpretations, we only need one head that captures a sense of directionality, or the lack thereof (cf. Kracht 2002; Romeu 2014). The import of this choice will become clear when we will address the semantic problem in section 4.2.

Overall, these structures suggest that our approach finds parallel analyses in the literature, but also that it seems to offer a more fine-grained and accurate analysis of the data than any previous proposals. Therefore, our approach seems to be already on the right track, while cartographic approaches face at least three problems. First, the flexible distribution of simple ISPs in either a lower or a higher position would render problematic any assumption about their status as either Kase or Place heads (or Aspect heads, as in the analysis of Tortora 2008). Second, this distributional flexibility would be problematic once we look at their semantic interpretation, since we would lack a principled reason to select the relevant interpretation (cf. Svenonius 2008). Third, the polymorphic distribution of simple ISPs as affix-like elements or relational heads would require the existence of empty specifier objects, in any analysis. Although less critical than the 
first and second problems, this approach disappears in our analysis, as we can freely connect these distributional patterns via the residuation rule.

Two further arguments based on our top-down approach offer further support for our analysis. First, in our approach we do not need to postulate movement operations that raise the inverted elements in topical/inverted position. ISPs such as di fronte and di fronte alla scrivania can be merged in situ, since their status as phrasal elements guarantees that the derivation proceeds straightforwardly. Second, we can block several partial derivations that would yield unattested strings, such as * fronte alla finestra or * di me e lui 'of me and him', which would be possible in these and other bottom-up approaches. Overall, since we have a solution to each of our three problems, we move to the semantic side of our analysis, and our fourth solution.

\section{The proposal: Semantic assumptions and analyses}

The goal of this section is to present our situation semantics apparatus (section 4.1), then discuss how this approach solves our fourth problem (section 4.2).

\subsection{The proposal: A Situation Semantics analysis}

The vast literature on the semantics of SPs offers many proposals on the ontological status of their denotations. Proposals include regions (Nam 1995; Kracht 2002), vectors (Zwarts \& Winter 2000), paths or events for directional SPs (Jackendoff 1983; 1990; Zwarts 2005; Landman 2000; Rothstein 2004; 2008a, respectively), or combinations of thereof (Krifka 1998; Gehrke 2008). These ontological distinctions are not mutually exclusive, though. For instance, Zwarts and Winter (2000) discuss how vectors can be conceived as ordered pairs of points in space, while Kracht (2002) suggests that vectors are best treated as sequences of points. Hence, these proposals seem to represent related alternatives converging to a unified approach to the semantics of SPs. For our purposes, a unified ontological approach is desirable, as we only need to discuss how the elements in the denotations of SPs interact with those in the denotation of other constituents.

For this purpose, we assume that all our morphemes denote elements belong to a universal set situations, regardless of whether these situations correspond to implicit or explicit referents. This set of situations forms a Boolean algebra, a partially ordered set with an empty situation (Landman 1991; Szabolcsi 2010, ch. 1), and with various sub-types of referents, such as individuals or spatial entities (Fintel 1994, ch. 2; Kratzer Acta Linguistica Hungarica 62, 2015 
2007). We take that a subset $L$ of spatial situations ("Locations") can be identified, and conceive locations as denoting three sub-types of sets of points (Bohnemeyer 2012): sequences of points ("vectors"); unordered sets of points ("regions"); temporally oriented sets of points ("paths"). We discuss our examples by appealing to either type or label, depending on which notion can better help us in illustrating the data at hand. ${ }^{10}$

We represent this domain as the set $S$, which includes a denumerably infinite set of elements (i.e., we have $S=\{\emptyset, s, r, t, v, \ldots, z\}$ ). When necessary, we use or drop subscripts for situations: $s$ is to be interpreted as a spatial situation or location $s_{l}$. We use "Quine's innovation", and assume that singleton sets represent atomic situations, i.e., $s$ stands for $\{s\}$ (Schwarzschild 1996, ch. 1), while complex sets represent sum situations (e.g., $\{s, g\}$ ). The set of types for situations is recursively generated by the definitions in (61):

(61) 1. Given a set $S$, a is a semantic type

(Lexical type)

2. If $a$ is a type and $b$ is a type, then $a \rightarrow b$ is a type

(Functional type)

3. If $a$ is a type and $b$ is a type, then $a \times b$ is a type

(Cartesian product type)

4. If $a \rightarrow b$ is a type and $b$ is a type, then $(a \rightarrow b) \times a=b$

(F. application)

5. If $a \rightarrow b$ is a type and $b \rightarrow c$ is a type, then $(a \rightarrow b) \times(b \rightarrow c)=a \rightarrow c$

(F. comp.)

6. Nothing else is a type

(Closure property)

In words, rule 1 defines the basic semantic type of situations, while rules 2 and 3 define more complex types via function abstraction and the (Cartesian) product type, denoted as " $\times$ " The product type should be interpreted as forming an ordered pair of values (in this case, situations), when the corresponding features are bundled together. Rules 4 and 5 show how these types interact via function application and function composition, the semantic counterparts of the forward application and cut rule (Landman 1991, ch. 2; Moortgat 2011, §2.2-2.3). We represent these semantic ternary relations via " =", as no confusion should arise between this symbol and its instances that denote identities between referents. Rule 6 says that no other operations can form and derive types. A set of types generated by these definitions is the set TYPE $=\{s, s \rightarrow s, s \rightarrow(s \rightarrow s), s \times s, \ldots\}$, which includes at least the types of referents, functions, relations and features' sets/properties. More complex types can be defined; for our purposes, these will suffice.

${ }^{10}$ Our ontology is closer to classic version of situation semantics (Barwise \& Perry 1999), than to its modern versions (e.g., Kratzer 2007; Elbourne 2013). This aspect is not crucial, here. 
As in the case of our morphological rules, we define a rule on types that allows to interpret the residuation rule, defined as: if $a \times b$ is a type and $c$ is a type, then $(a \times b) \rightarrow c=a \rightarrow(b \rightarrow c)$. This rule introduces a relation between application and composition as a "Residuation Interpretation" principle (RI), an operation that allows the very constrained suppression or promotion of arguments in functions and relations.

We now turn to interpretive matters. We implement a simple form of $\boldsymbol{\lambda}$-calculus to represent our functions and relations, and define the part-of relation as the main relation holding between situations. This relation is usually represented as " $a \leq b$ ", which reads: " $a$ is part of $b$ ". The following properties hold: if $a$ is part of $b$, then $(a \wedge b)=a$ and $(a \vee b)=b$. In other words, if a situation $a$ is part of a situation $b$, then their intersection will correspond to $a$, the subset situation, and their union to $b$, the super-set situation. As we implement Quine's innovation for our situations, mereological sum (product) and set union (intersection) reduce to the same operations.

The part-of relation allows us to establish a general partial order amongst situations: the precise types of situations at stake define more specific properties of this relation. For locations, the part-of relation is interpreted as a "coordinate" relation. This is the case, since this relation establishes that a certain location is part of a topological space defined with respect to the ground (cf. Nam 1995; Zwarts \& Winter 2000; Bohnemeyer 2012). Thus, we treat the semantics of ISPs as involving relations between locations, defined as regions of space, or parts thereof. For our purposes, this more coarse-grained definition of the part-of relation, and its ability to instantiate a situation when saturated (i.e., when $\lambda$-conversions are made), will suffice.

For practical reasons, we use a prefixed notation for the part-of relation, i.e., $P(a, b)$. This allows us to represent complex or structured situations simply as: " $\lambda x . \lambda y . s: P(x, y)$ ", a notation we import from DRT's treatment of events and other related frameworks (von Fintel 1994; Land-

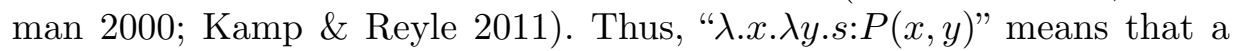
situation is defined as, or instantiated by, a relation between two referents, yet to be specified (cf. also von Fintel 1994, ch. 1). This notation is a shorthand for $\lambda . x . \lambda y \cdot \exists s .[P(x, y, s)]$, the type of notation commonly found in event/situation semantics works (Rothstein 2004; Kratzer 2007), but our notation has the advantage of being slightly more "compact". For the time being, we treat $s$ as a free variable, as we will specify how these variables get bound when we will discuss our data in detail. 
We now define the relation between our morphological and semantic types, via an isomorphism defined as a general instance of the interpretation function. For instance, the result of merging a unit of type $\boldsymbol{p} / \boldsymbol{p} / \boldsymbol{p}$ with two $\boldsymbol{p}$ units is a Phrase of a "recursive" $\boldsymbol{p}$ type that denotes a structured situation $s$. We show this mapping in (62):

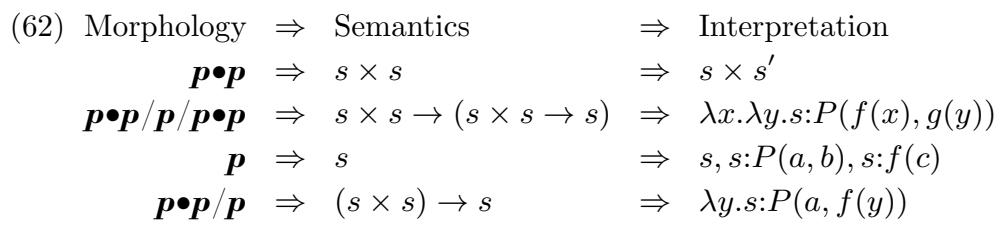

These interpretations read as follows. Features (type $\boldsymbol{p} \bullet \boldsymbol{p}$ ) can find their denotation in the product type $s \times s$ of situations, and can denote ordered situations. Relations carrying features on their arguments (type $\boldsymbol{p} \bullet \boldsymbol{p} / \boldsymbol{p} / \boldsymbol{p} \bullet \boldsymbol{p})$ denote objects of type $s \times s \rightarrow(s \times s \rightarrow s)$, the type of part-of relations that have specific restrictions as to what arguments can be in their domain (here: $\lambda x . \lambda y . s: P(f(x), g(y))$ ). The functions $f$ and $g$ represent the possible restrictions on the types of argument on the type of arguments (Landman 1991, ch. 2; Harbour 2007, ch. 2; Szabolcsi 2010, ch. 1). Phrases (type $\boldsymbol{p}$ ) denote situation types $s$, the types of referents and those of saturated relations and functions. Affix-like elements (type $\boldsymbol{p} \boldsymbol{p} / \boldsymbol{p})$ denote situation types $(s \times s) \rightarrow s$, the type of functions/properties or partially saturated relations with a restriction over their argument.

We can now offer a semantic derivation of (51) in (63) to illustrate how these principles work, presenting the interpreted morphemes with the operations interpretation (Int) and function application/composition (FA/FC), as semantic matches of LS and MI:

(63) a. Mario loves Peach.

$$
\begin{aligned}
& \text { b. t. } \llbracket \text { Mario } \mathbb{p} \rrbracket=m \\
& \mathrm{t}+1 \text {. } \llbracket \text { loves }_{\boldsymbol{p} / \boldsymbol{p} / \boldsymbol{p}} \rrbracket=\lambda x \lambda y . s: l(x, y)_{s \rightarrow(s \rightarrow s)} \\
& \mathrm{t}+2 . \llbracket \boldsymbol{p} / \boldsymbol{p}\left[\operatorname{Mario}_{\boldsymbol{p}}\right] \operatorname{loves}_{\boldsymbol{p} / \boldsymbol{p} / \boldsymbol{p}} \rrbracket=\lambda x \lambda y \cdot s: l(x, y)_{s \rightarrow(s \rightarrow s)}\left(m_{s}\right)= \\
& =\lambda y \cdot s: l(m, y)_{s \rightarrow s} \\
& \mathrm{t}+3 \cdot \llbracket \text { Peach } \boldsymbol{p} \rrbracket=p_{s} \\
& \mathrm{t}+4 . \llbracket \boldsymbol{p}\left[\text { Mario }_{\boldsymbol{p}}\right] \operatorname{loves}_{\boldsymbol{p} / \boldsymbol{p} / \boldsymbol{p}}\left[\operatorname{Peach}_{\boldsymbol{p}}\right] \rrbracket=\lambda y . s: l(m, y)_{s \rightarrow s}\left(p_{s}\right):=s_{s}: l(m, p)
\end{aligned}
$$

In (63), "โ.』" represents the interpretation function. For the sake of simplicity, we translate loves as a relation $s: l(x, y)$, a(n unbounded) situation in which two referents stand in a "love" relation. Thus, the interpretations of Mario and Peach, the referents $m$ and $p$, become the arguments of the 
love relation $l$ in this order, via standard function application. This interpretation is obtained as soon as the morphosyntactic structure is derived, in a step-wise fashion. We now turn to our data.

\subsection{The semantic analysis: The data}

We start our analysis by rehearsing some basic facts about the distribution of our ISPs with respect to aspect and specificity. Recall that an ISP such as dietro alla 'behind' can denote a non-specific location, while dietro $(P)$ $l a$ denotes a specific location. Specificity, defined as the ability of noun or determiner phrases to denote referents that are known in discourse, interacts with lexical aspect, as seen in (27)-(31). Thus, if we want to account for our data, then we must distinguish the two dimensions of meaning. At a morphological level, notions such as specificity or (un)boundedness correspond to features qua compound types. Our problem, however, lies in establishing at what semantic level the corresponding senses work, and how they can be defined.

Most approaches to lexical aspect contend that notions such as cumulativity can better capture whether a predicate is unbounded or not. Unbounded predicates have cumulative denotation, whereas bounded predicates lack this property (Krifka 1998; Rothstein 2004; Zwarts 2005). Predicates, in turn, have a cumulative denotation when both atomic and nonatomic objects are part of their denotation. We discuss a formal analysis of cumulativity via Krifka (1998) approach, presented in (64)-(65)

(64) $C M(X) \Leftrightarrow \forall x . \forall y . \forall z .(X(x, z) \wedge X(y, z) \rightarrow X(x \vee y, z))$

(65) $C M(s): X(x, z)) \Leftrightarrow C M(X)$

In (64), a relation, and the situation it instantiates, is cumulative if and only if a property holds for its atomic individuals, then it also holds for its (mereological) sum individuals.

A more compact definition is in (65): this identity says that cumulativity acts as a property of the situations in which a relation holds. Since situations, in our system, are instantiated by the sets of objects that belong to the domain of a relation, cumulativity is defined over a relation $X$ (i.e., we have $C M(X)$ ), and a relation corresponds to a situation ( $X$ stands for $s: P(x, y)$, a short-hand for $\exists s .[P(x, y, s)])$. Hence, the left-side of (65) is short-hand for $C M(s)$. [X(x,y,s)]. Given the equivalences in (64)-(65), cumulativity can be defined as the mapping of several more specific properties and corresponding situations (e.g., $\forall x . \forall y . \forall z .\left(s: X(x, z) \wedge s^{\prime}: X(y, z) \rightarrow\right.$ 
$\left.\left.s^{\prime \prime}: X(x \vee y, z)\right)\right)$. Thus, cumulativity can be treated as corresponding to a product type morpheme $\boldsymbol{p} \bullet \boldsymbol{p}$, as the identity in (65) indirectly states. It denotes an operator $C M$ that binds situation variables (cf. the $G E N$ operator in DRT: Kamp \& Reyle 2011), and constrains which situations can be in the denotation of a relation.

We turn our attention of specificity and its formalization. For this purpose, we take a perhaps simplistic perspective based on von Heusinger (2012), and assume that specificity amounts to a choice function $\boldsymbol{f}$ that selects a relevant entity in discourse, out of a set. We give a definition of a choice function $f$ in (66), and a situation-based formulation of specificity in (67) (cf. von Heusinger 2012, 1039):

(66) $f$ is a choice function: $\operatorname{ch}(f) \Leftrightarrow P(f(P))$

(67) $\operatorname{ch}(f) \Leftrightarrow s: P(f(s: P)), \lambda x \cdot \lambda y \cdot s: P(s c(x), y)$

The equivalence in (66) says that a choice function $f$ applies to any nonempty set $P$ and returns (chooses) one of its elements, which in turn is defined once more as belonging to $P$. For instance, if $P$ is a set of men, $f$ selects one specific element, and this specific element is individuated once more as a man. The equivalence in (67) says that a choice function that applies to a situation chooses a pair of locations (parts and ground) in the domain of the relation instantiating this situation. The function returns one of its elements, an ordered pair. This ordered pair is individuated once more as instantiating a relation that holds in a situation, but restricted to this specific pair. Although other instances of specificity would require other translations (cf. Von Heusinger 2012's discussion on the $\epsilon$-calculus), these translations suffice to distinguish cumulativity and specificity. For the definiteness feature on definite articles, we make the standard assumption of using the $\iota$-operator, which suffices for our purposes (Heim 2012).

We can now focus on the underspecified interpretation of ISPs with respect to lexical aspect/cumulativity. If compound types represent product sets of features, then underspecified features can be represented as so-called "co-product" or "disjoint" types (Moortgat 2010, §2; Morrill 2011, ch. 2) ${ }^{11}$ In our case, we represent under-specified values as union sets of situations: $\vee\{+X(s),-X(s)\}$, or $\pm X(s)$ for short (cf. Harbour 2007; Harley 2012). While positive marking amounts to identity (i.e., $+X(s)=X(s)$ ),

${ }^{11}$ We have not defined this type constructor in thorough detail, since it plays such a very limited role, so we can only afford to focus on its semantic reflection. See the cited literature for discussion. 
negative marking amounts to negation of a property: we thus have the identity $-X(s)=\neg X(s)$. The semantic type assignment in (68), based on the mapping in (53), and the interpretation of relevant lexical items in (69) are the final step we need to account our data:

(68) a. $s \times s::=\{l$ lui, davanti, dietro, sopra, di fronte, $(p), \ldots\}$

b. $s \times s \rightarrow(s \times s \rightarrow s)::=\left\{d i, d e-\right.$, del, da, $(P),(P)^{\prime}$, al, alla,$\left.\ldots\right\}$

c. $s::=\{$ scrivania, tavolo, fronte, di fronte alla finestra,... $\}$

d. $s \times s \rightarrow s::=\{a, i l, l a, d i, n e,-l, \ldots\}$

(69) a. $\llbracket$ davanti $\rrbracket= \pm s: P(d v, g r(c)), \llbracket$ sopra $\rrbracket= \pm C M s: P(s p, g r(c))$

b. $\llbracket \mathrm{a} \rrbracket=\lambda x \cdot \lambda y \cdot \pm C M(s): P(-s c(x), \operatorname{gr}(y))$, $\llbracket(\mathrm{P}) \rrbracket=\lambda x \cdot \lambda y \cdot \pm C M(s): P(+s c(x), \operatorname{gr}(y))$, $\llbracket \mathrm{va} \rrbracket=\lambda x \cdot \lambda y \cdot-C M(s): \boldsymbol{g o}^{\prime}(x, y)$, $\llbracket \mathrm{di} \rrbracket=\lambda x \cdot \lambda y \cdot \pm C M(s): P( \pm s c(x), \operatorname{gr}(y))$

c. $\llbracket$ fronte $\rrbracket=f r, \llbracket$ scrivania $\rrbracket=s v, \llbracket$ divano $\rrbracket=d v$, $\llbracket \operatorname{mezzo} \rrbracket=m z, \llbracket \operatorname{stanza} \rrbracket=s t$

d. $\llbracket \mathrm{la} \rrbracket=\lambda y \cdot s: \iota(y), \llbracket \mathrm{il} \rrbracket=\lambda y \cdot s: \iota(y), \llbracket \mathrm{di} \rrbracket=\lambda x \cdot s: P( \pm s c(x), g r(c))$

In (69a), projective ISPs that can occur "bare" (e.g., davanti 'ahead') denote product situations: saturated relations between locations. Simple ISPs that act as relational elements ( $d i$ in (69b)) denote relations with restrictions on their arguments: their arguments are spatial situations/locations. The function/restriction ground thus changes the sub-type of a referent (e.g., an individual) into a spatial type situation (Svenonius 2008; and our (49)). For reasons of space, we drop this restriction in our derivations, as it will become clear in (70) and (71). In the case of $a$ 'at, to' as a specificity marker, we assume that it marks its first argument as non-specific (i.e., $-s c(x)$ ), while "(P)" marks it as specific (i.e., $+s c(x)$ ), and $d i$ as underspecified (i.e., $\pm s c(x))$. DPs, "non-bare" projective ISPs (e.g., fronte 'front') and full ISP phrases are assigned type $s$ (viz. (69c), and are mapped onto location types once they merge with other items (e.g., $d i$ in di fronte 'in front', scrivania 'desk' as a ground DP). Affix-like items (e.g., di in di fronte) are assigned type $s \times s \rightarrow s$ and can include a restriction on their argument (viz. (69d)). Via the residuation rule, these affixes also include a saturated/suppressed argument, the ground $g r(c)$, in their denotation, and made explicit once the full ISP phrase is derived.

We make these assumptions clear via our first derivation. Morphological types and derivations are dropped; interpretation and function application/composition (Int, FA/FC respectively) are the semantic counterparts 
of our morphological operations. We first derive the interpretation of our ISPs in (70b), the semantic counterpart of (54b). Note: here onwards, we may omit "(FA)" markers in certain steps, for reasons of space:

(70) a. Mario siede di fronte alla scrivania.

'Mario sits in front of the desk.'

b. t. $\left.\llbracket \mathrm{di} \rrbracket=\lambda x \cdot \pm C M s^{\prime}: P( \pm s p(x), c)\right)_{s \rightarrow s} \quad$ (Int)

$t+1$. $\llbracket$ fronte $\rrbracket=f r_{s}$

$t+2 . \llbracket \mathrm{di} \rrbracket(\llbracket$ fronte $\rrbracket)=\lambda x . \pm C M s^{\prime}: P( \pm s c(x), c)_{s \rightarrow s}\left(f r_{s}\right)=$ $= \pm C M s^{\prime}: P( \pm s c(f r), c)_{s}$

$t+3 . \llbracket \mathrm{a} \rrbracket=\lambda x \cdot \lambda y \cdot \pm C M s: P(-s c(x), y))_{s \rightarrow(s \rightarrow s)}$

$t+4$. (【di fronte $\rrbracket) \llbracket \mathrm{a} \rrbracket=$

$=\lambda x \cdot \lambda y \cdot( \pm C M s: P(-s c(x), y))))_{s \rightarrow(s \rightarrow s)}\left( \pm C M s^{\prime}: P( \pm s c(f r), c)\right)_{s}=$

$=\lambda y \cdot \pm C M s: P\left( \pm C M s^{\prime}: P(-s c(f r), c), y\right)_{s \rightarrow s}$

$t+5 . \llbracket 1 \mathrm{a} \rrbracket=\lambda y . \iota(y)_{s \rightarrow s}$

$t+6$. $(\llbracket$ di fronte $\mathrm{a} \rrbracket)(\llbracket 1 \mathrm{la} \rrbracket)=$

$=\left(\lambda y \cdot \pm C M s: P\left( \pm C M s^{\prime}: P(-s c(f r), c), y\right)\right)_{s \rightarrow s}\left(\lambda y \cdot \iota(y)_{s \rightarrow s}\right)=$

$=\lambda y \cdot \pm C M s: P\left( \pm C M s^{\prime}: P(-s c(f r), c), \iota(y)\right)_{s \rightarrow s}$

$t+7$. scrivania $\rrbracket=s v_{s}$

$t+8$. 【di fronte alla $\rrbracket(\llbracket$ scrivania $\rrbracket)=$

$=\lambda y \cdot \pm C M s: P\left( \pm C M s^{\prime}: P(-s c(f r), c), \iota(y)\right)_{s \rightarrow s}(s v)_{s}=$

$= \pm C M s: P(-s c(f r), \iota(s v))_{s}$

The derivation in (70b) reads as follows. The constituent di fronte is interpreted as a situation in which a frontal part is defined with respect to an implicit ground $c$ (steps $t$ to $t+2$ ). When $a$ is merged, it also identifies the resulting situation as being non-specific and ambiguous with respect to aspect (steps $t+3, t+4$ ). When $l a$ is merged, it forms a single constituent with $a$ (i.e., we have alla steps $t+5, t+6$ ), with the property of definiteness acting as a restriction on the incoming ground argument (steps $t+7, t+8)$. Thus, di fronte alla scrivania 'in front of the desk' denotes a non-specific position (the $-s c(f r)$ part) defined as belonging to the frontal axis of the desk (the part-of relation that $P$ denotes). This result mirrors on the semantic side, via function composition, how the cut rule can bundle features together and remove structure.

Two important observations are due. First, both $d i$ and $a$ introduce situations that are bound by the (underspecified) $\pm C M$ operator. This means that the relation di fronte alla scrivania 'in front of the desk' may have cumulative (non-cumulative) denotation, but also that the complex 
SP di fronte 'in front' may have cumulative (non-cumulative) denotation. In words, di fronte denotes both atomic and sum locations that qualify as being "in front", and these are part of the space defined with respect to the desk, which includes atomic and sum locations. Thus, the two cumulativity operators do not interact. Second, the merge of scrivania 'desk' establishes an explicit value $s v$ for the referent qualifying as the ground. Thus, the identity $c=\iota(s v)$ can be established (or more accurately, $+\operatorname{gr}(c)=\operatorname{gr}(\iota(s c)))$, which also allows to identify the situations $s$ and $s^{\prime}$ (the two part-of relations are identical), and simplify the denotation (step $t+8$ ). This identification procedure is similar to treatments of anaphora resolution in other situation semantics approaches (Elbourne 2013), or in DRT (Kamp \& Reyle 2011). This fact will play a role in the argument demotion analysis.

The main net result is that we can show how di fronte alla scrivania denotes a situation in which a frontal, non-specific position for the figure is obtained in an entirely compositional manner. We now turn to the interpretation of our example (58), repeated here as (71). We remove non-necessary brackets, when possible, and type sub-scripts:

(71) a. Mario siede nel mezzo della stanza.

'Mario sits in the middle of the room.'

b. $t . \quad \llbracket \mathrm{ne} \rrbracket=\lambda x \cdot \pm C M s^{\prime}: P( \pm s c(x), c)$

$t+1 . \llbracket \mathrm{il} \rrbracket=\lambda x . \iota(x)$

$t+2 . \quad \llbracket$ ne $\llbracket \mathrm{il} \rrbracket=\lambda x . \pm C M s^{\prime}: P( \pm s c(x), c)(\lambda x . \iota(x))=$ $=\lambda x . \pm C M s^{\prime}: P( \pm s c(\iota x), c)$

$t+3$. mezzo $\rrbracket=m z_{s}$

$t+4$. $\llbracket$ nel $\rrbracket(\llbracket$ mezzo $\rrbracket)=\lambda x . \pm C M s^{\prime}: P( \pm s c(\iota x), c)(m z)=$ $= \pm C M s^{\prime}: P( \pm s c(\iota m z), c)$

$t+5 . \llbracket \operatorname{de} \rrbracket=\lambda x \cdot \lambda y \cdot \pm C M s: P( \pm s c(x), y)$

$t+6 . \quad(\llbracket$ nel mezzo $) \llbracket \mathrm{de} \rrbracket=$

$=\lambda x \cdot \lambda y \cdot \pm C M s: P( \pm s c(x), y)\left( \pm C M s^{\prime}: P( \pm s c(\iota m z), c)=\right.$ $=\lambda y \cdot \pm C M s: P\left( \pm C M s^{\prime}: P( \pm s c(\iota m z), c), y\right)$

$t+7 . \llbracket-\mathrm{a} \rrbracket=\lambda y \cdot \iota(y))_{s \rightarrow s}$

$t+8 . \llbracket$ nel mezzo de $\rrbracket(\llbracket-\mathrm{la} \rrbracket)=$

$=\lambda y \cdot \pm C M s: P\left( \pm C M s^{\prime}: P( \pm s c(\iota m z), c), y\right)(\lambda y \cdot \iota(y))=$ $=\lambda y \cdot \pm C M s: P\left( \pm C M s^{\prime}: P( \pm s c(\iota m z), c), \iota y\right)$

$t+9 . \llbracket$ stanza $\rrbracket:=s t_{s}$ 


$$
\begin{aligned}
& t+10 \text {. } \\
& =\lambda y \cdot \pm C M s: P\left( \pm C M s^{\prime}: P( \pm s c(\iota m z), c), \iota y\right)(s t)= \\
& = \pm C M s:( \pm s c(\iota m z), \iota s t)
\end{aligned}
$$

The crucial aspects of this derivation are as follows. The ISP nel mezzo della stanza a situation in which the central part/location of the room is taken as the definite position for the ground, whether it be specific or nonspecific (steps $t$ to $t+4$ ). Cumulativity then establishes that this more complex location can also be made of "smaller" locations, such as those including the central location, or more complex locations (steps $t+5$ to $t+8)$. Thus, the interpretation of this ISP phrase follows the same derivational processes that compute the interpretation of di fronte alla scrivania, modulo the small differences corresponding to the different lexical items making up this ISP (i.e., nel mezzo).

We continue our analysis of the data by analysing how our aspectually underspecified ISPs can merge with verbs and become unambiguous. Since we treat underspecified terms as union sets, point-wise function application yields multiple "alternative" values. A function yields a result for each of the possible values that its argument can take. In the case of ISPs and verbs, this may result in one or more results being uninterpretable, as two operators of opposite polarity may bind the same situation variable. We offer a simplified derivation in (72) to illustrate this point:

(72) a. Mario va sopra il divano. 'Mario goes over the sofa.'

b. $\quad k . \quad \llbracket \mathrm{va} \rrbracket=\lambda y \cdot-C M s: \mathbf{g o} \boldsymbol{o}^{\prime}(m, y)$

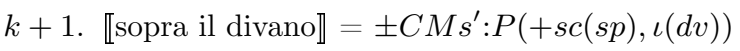

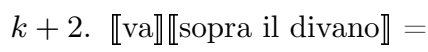

$$
\begin{aligned}
= & \lambda y .-C M s: \mathbf{g o} \boldsymbol{o}^{\prime}(m, y)\left( \pm C M s^{\prime}: P(+s c(s p), \iota(d v))\right)= \\
=\left\{-C M s: \mathbf{g o}{ }^{\prime}\left(m,-C M s^{\prime}: P(+s c(s p), \iota(d v)),\right.\right. & \\
& -C M s: \mathbf{g o} \boldsymbol{o}^{\prime}\left(m,\left(+C M s^{\prime}: P(+s c(s p), \iota(d v))\right)=\#\right\}
\end{aligned}
$$

Our simplified derivation reads as follows. First, va 'goes' merges with the ISP phrase sopra il divano (steps $k$ to $k+2$ ). Since this ISP phrase is ambiguous (underspecified) between a cumulative and non-cumulative reading, two possible interpretations arise, as the final passage of $k+2$ shows. In the non-cumulative interpretation, Mario goes to the sofa and reaches the "over" location (i.e., $-C M s^{\prime}$, in compressed form). According to the cumulative interpretation, Mario goes towards the sofa, but may reach 
locations that would only partially be over the sofa. This structural incongruity creates an uninterpretable sentence, something that we represent via the symbol "\#". Thus, we can account that only the non-cumulative interpretation is accessible, in these cases, as previously observed in the literature (cf. Fong 1997; Zwarts 2005; 2008; Egg 2011).

Overall, our approach can offer a compositional account of our data. Also, it can be extended to cover other anaphoric patterns: pronominal ground DPs (e.g., lui 'him') and the interpretation of argument demotion and locative inversion data. Recall from sections 2.4 and 3.1: if the ground DP is a pronoun, an overt simple ISP ( $d i$ or $d a$, usually) must be phonologically realised, even with ISPs that normally resist this insertion. We repeat $(19) /(59)$ as $(73)$, and (32) as (74):

(73) Mario è dietro di $/ *(\mathrm{P})$ lui.

'Mario is behind him.'

(74) Mario è da Luigi.

'Mario is at Luigi's (place).'

A simple analysis is that $d i$, but not the silent "(P)" head, carries the relevant property that restricts the interpretation of lui 'him' accordingly. Since these pronouns denote animate referents, rather than locations, $d i$ must act as a case marker-like element that interprets them as grounds. This assumption also predicts that coordinated pronouns must involve the repetition of simple ISPs. One example is (22), in which we have Mario va verso *di me o lui/di me o di lui 'Mario goes towards me or him'. If a pronoun must be marked as a ground DP, then each pronoun in a sentence must merge with a corresponding simple ISP, before becoming part of a coordinated structure. The upshot of this analysis is that, once we capture how ISPs impose selectional, restrictions on their arguments, the context-sensitive distribution of simple ISPs is accounted for.

By this point, we can also formulate a straightforward semantic account of argument demotion cases. We repeat (11) as (75) to discuss our analysis:

(75) Luigi é di fronte al tavolo da biliardo. Mario é dietro (al tavolo da biliardo). 'Luigi is in front of the billiard pool. Mario is behind (at-the billiard pool).'

For a full account, we would need to extend our analysis beyond the sentence level, a task we leave for future research (but see Jäger 2001; Ursini 2015). Given our ability to account for (basic) anaphoric relations, however, can offer a preliminary analysis. At some point in a derivation, we 
have the situation $s: P(-s c(f r), c)$. The (partial) identity $g r(c)=g r(t v)$ between ground locations can be established ('behind the implicit ground, identified as the table'). When this identity is established, the phonological component "demotes" the second occurrence of the simple ISP introducing the ground DP. Hence, the interpretation of the demoted ground is anaphorically given, as per predictions and standard assumptions on ellipsis (Merchant 2001; 2004; Jäger 2005).

We can now outline a simple account for locative inversion cases. Although a full-fledged treatment of locative inversion would need a discussion of topics such as focus and information structure, we know that its core semantic effect is that of making the inverted ISP "topical" in discourse. We repeat $(41) /(60)$ as (76), to capture our analysis:

(76) Di fronte alla finestra, I ragazzi bevono birra.

'In front of the window, the boys drink beer.'

We can assume that a silent " $(\mathrm{P})^{\prime \prime}$ " head takes the inverted ISP phrase and the full clause I ragazzi bevono birra as its arguments. Its interpretation can then determine that the phrase di fronte alla finestra denotes the location in which the situation involving boys that drink beer takes place as being topical in discourse. We assume that the ISP phrase denotes the situation $s$ and that the clause $I$ ragazzi... denotes the situation $s^{\prime}$. The silent element " $(\mathrm{P})^{\prime \prime}$ " denotes the relation $\lambda x . \lambda y . s^{\prime \prime}: l o c:(x, y)$ which, once saturated, becomes the situation $s^{\prime \prime}: l o c:\left(s, s^{\prime}\right)$ : the ISP phrase denotes the (topical) location of a situation $s^{\prime}$.

Overall, we have a clear, transparent mapping between the two levels of analysis, which displays a typical form of "direct compositionality" typical of TLS. Therefore, we can conclude that we have offered a solution to our fourth problem.

\subsection{The semantic analysis: A discussion}

As our discussion in the previous discussion has shown, our semantic analysis bears more than one resemblance to the analyses we discussed in section (2.4.2), one example being Svenonius (2008). Given these similarities, we compare our (full) interpretation for the ISP phrase di fronte alla scrivania 'in front of the desk' offered in (51), and repeated here as (77a), with $(49 \mathrm{c})$, repeated as $(77 \mathrm{~b})$ :

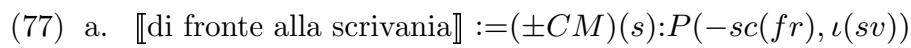

b. 【in front of the house $\rrbracket:=\lambda V . \exists l \exists l^{\prime}\left[\operatorname{project}\left(V, l^{\prime}\right) \wedge \operatorname{front}-\operatorname{part}\left(l, l^{\prime}\right) \wedge \operatorname{Eigen}\left(h, l^{\prime}\right)\right]$ 
In words, (77a) captures an aspectually underspecified situation in which a relation with a unique ground, a desk $s c$, and an unspecified frontal position holds. The logical form in (77b) denotes approximately the same relation, although in a less compact manner, and without addressing specificity and lexical aspect. Importantly, this form also introduces several locations and vectors as referents that can become elements bound in anaphoric relations, such as those licensed when argument demotion occurs. This result seems to be problematic with respect to our ISP data, much like the lack of a treatment of specificity and lexical aspect, with two problems being particularly relevant.

First, an extension of the analysis in (77b) to pronoun-based data (i.e., (73)-(74)) would require a theory on which simple ISP is selected, and why. Since simple ISPs can occur in what we have defined as either Place or Kase positions, and since their exact interpretation depends on their syntactic position, this task is far from trivial. We would have $d i$, to either (partially) denote the relation "project $\left(l, l^{\prime}\right)$ " or the relation "Eigen $\left(x, l^{\prime}\right)$ ", with the first option failing to capture the fact that lui is marked as a ground DP. Second, once we consider locative inversion and argument demotion data, this proliferation of referents as possible antecedents (i.e., $l^{\prime}, l$ or $V$ ) makes the resolution of anaphoric relations problematic. Since it is problematic to establish which referent is marked as a ground, it would be problematic to establish precise conditions on when argument demotion and, indirectly, locative inversion are licensed.

These problems are not beyond the reach of this approach, provided that one would update it with the relevant assumptions to account for the data. However, Svenonius (2008) already improves over previous syntactic analyses of ISPs (Tortora 2008; Folli 2008) and over non-compositional analyses of SPs in general (e.g., Nam 1995; Zwarts \& Winter 2000). Hence, our approach seems to offer an analysis of ISPs that is more accurate than all previous proposals, and apparently involves fewer assumptions. In doing so, we also show that our proposal is consistent with other proposals on SPs in the literature (e.g., Hale \& Keyser 2002; Zwarts 2005; 2008). We therefore conclude that we have solved our four problems about ISPs successfully, and can now move to the general conclusions.

\section{Conclusions}

In this paper we have presented an approach of Italian spatial prepositions that presents novel data and improves over previous proposals (Rizzi 1988; Tortora 2005; Folli 2008). We have shown that different types of 
ISPs, such as a 'at/to', sopra 'above', di fronte 'in front', verso 'towards' can all receive the same morphological analysis from which a principled semantic analysis can be derived straightforwardly. This analysis covers types of ISPs, syntactic patterns and semantic phenomena not discussed in the previous literature (multi-morphemic ISPs: di fronte; locative inversion, argument demotion; selectional restrictions; RS; specificity and lexical aspect). As our analysis suggests, all these phenomena can receive a fully compositional treatment that would be possible only via supplementary assumptions, in other approaches. Overall, our proposal seems to correctly capture the data in (1)-(43) and, in doing so, it extends TLS, DM and situation semantics approaches to cover these morpho-semantic phenomena.

We conclude by observing that a broader set of data, whether they involve ISPs or related data (e.g., spatial adverbs and prefixes), could be analysed within our approach. A well-known fact is that in German, among other languages, SPS and case markers interact in interesting ways (Kracht 2002; Riemsdijk \& Huybregts 2007). Several SPs can receive either a locative or a directional interpretation, depending on whether they merge with a dative- (locative) or accusative-marked (directional) ground DP. It is our conjecture that the analysis we developed to account for the context-sensitive distribution of $a$ and $d i$, as well as their semantic underspecification, can be extended to this type of data. However, in such a case we would move beyond the topic of ISPs proper. For this reason, we leave these topics for future research.

\section{Acknowledgements}

Many thanks to three anonymous reviewers for the important and useful suggestions; to Aijun Huang, and Ivano Caponigro for feedback; and to my princess for support. The usual disclaimers apply.

\section{References}

Absalom, Matthew and John Hajek. 2006. Prosodic phonology and raddoppiamento sintattico: A re-evaluation. In K. Allan (ed.) Proceedings of the 2005 Conference of the Australian Linguistics Society. Melbourne: Monash University. 1-14.

Adger, David. 2010. A minimalist theory of feature structure. In A. Kibort and G. Corbett (eds.) Features: Perspectives on a key notion in linguistics. Oxford: Oxford University Press. 185-218.

Asbury, Anna. 2008. The morphosyntax of case and adpositions. Doctoral dissertation. University of Utrecht. 
Asbury, Anna, Jacub Dotlačil, Berit Gehrke, Øystein Nilsen and Rick Nouwen (eds.). 2008. Syntax and semantics of spatial P. Amsterdam \& Philadelphia: John Benjamins.

Barwise, Jon and John Perry. 1999. Situations and attitudes. Second edition. Cambridge, MA: MIT Press.

Bohnemeyer, Jurgen. 2012. A vector space semantics for reference frames in Yucatec. In E. Bogal-Allbritten (ed.) Proceedings of the Sixth Meeting on the Semantics of UnderRepresented Languages in the Americas (SULA 6) and SULA-Bar. Amherst, MA: GLSA Publications. 15-34.

Cinque, Guglielmo and Luigi Rizzi (eds.). 2010. Mapping spatial PPs: The cartography of syntactic structure. Volume 6. New York: Oxford University Press.

Cresswell, Maxwell J. 1978. Prepositions and points of view. Linguistics and Philosophy 2. $1-41$

Dikken, Marcel den. 2006. Relators and linkers: The syntax of predication, predicate inversion, and the copula. Cambridge, MA: MIT Press.

Dikken, Marcel den. 2010. On the functional structure of locative and directional PPs. In Cinque \& Rizzi (2010, 74-126).

Dowty, David R. 1989. On the semantic content of the notion 'thematic role'. In G. Chierchia, B. H. Partee and R. Turner (eds.) Properties, types and meanings. Vol. II: Semantic issues. Dordrecht: Kluwer. 69-130.

Egg, Marcus. 2011. Underspecification. In Maienborn et al. (2011, 533-572).

Elbourne, Paul. 2013. Definite descriptions. Oxford: Oxford University Press.

Embick, David and Rolf Noyer. 2001. Movement operations after syntax. Linguistic Inquiry 32. 555-95.

Embick, David and Rolf Noyer. 2006. Distributed morphology and the syntax/morphology interface. In G. Ramchand and C. Reiss (eds.) The Oxford handbook of linguistic interface. Oxford: Oxford University Press. 305-324.

Emonds, Joseph. 1985. A unified theory of syntactic categories. Dordrecht: Foris.

Fábregas, Antonio. 2007. Axial parts and wholes. Nordlyd Troms $\varnothing$ Working Papers on Language \& Linguistics 34. 1-32.

Fintel, Kai von. 1994. Restrictions on quantifier domains. Doctoral dissertation. University of Massachusetts at Amherst.

Folli, Raffaella. 2002. Constructing telicity in English and Italian. Doctoral dissertation. University of Oxford.

Folli, Raffaella. 2008. Complex PPs in Italian. In Asbury et al. (2008, 197-221).

Folli, Raffaella and Gillian Ramchand. 2005. Prepositions and results in Italian and English: An analysis from event decomposition. In H. J. Verkuyl, H. de Swart and A. van Hout (eds.) Perspectives on aspect. Dordrecht: Springer. 81-105.

Fong, Vivienne. 1997. The order of things: What directional locatives denote. Doctoral dissertation. Stanford University.

Franco, Ludovico and Elsa Zampieri. 2012. Against a lexical account of inflected prepositions in Italian: Experimental evidence from aphasia. ReVEL 10. 50-62.

Frascarelli, Mara. 2000. The syntax-phonology interface in focus and topic constructions in Italian. Dordrecht: Kluwer. 
Gehrke, Berit. 2008. Ps in motion: On the semantics and syntax of P elements and motion events (LOT Dissertation Series 184). Utrecht: Landelijke Onderzoekschool Taalwetenschap.

Hale, Kenneth and Samuel Jay Keyser. 2002. Prolegomenon to a theory of argument structure. Cambridge, MA: MIT Press.

Harbour, Daniel. 2007. Morphosemantic number: From Kiowa noun classes to UG number features. Dordrecht: Springer.

Harley, Heidi. 2010a. Affixation and the mirror principle. In R. Folli and C. Ulbrich (eds.) Interfaces in linguistics: New research perspectives. Oxford: Oxford University Press. $166-186$.

Harley, Heidi. 2010b. A minimalist approach to argument structure. In C. Boeckx (ed.) The Oxford handbook of linguistic minimalism. Oxford: Oxford University Press. 426-447.

Harley, Heidi. 2012. Semantics in Distributed Morphology. In Maienborn et al. (2012, 688709).

Heim, Irene. 2012. Definiteness and indefiniteness. In Maienborn et al. (2012, 996-102).

Heusinger, Klaus von. 2012. Specificity. In Maienborn et al. (2012, 1025-1058).

Jackendoff, Ray. 1983. Semantics and cognition. Cambridge, MA: MIT Press.

Jackendoff, Ray. 1990. Semantic structures. Cambridge, MA: MIT Press.

Jäger, Gerhard. 2001. Anaphora and quantification in Categorial Grammar. In M. Moortgat (ed.) Logical aspects of computational linguistics. Third International Conference, LACL'98, Grenoble, France, December 1998, Selected papers (Springer Lecture Notes in Artificial Intelligence 2014). Springer: Berlin. 70-90.

Jäger, Gerhard. 2005. Anaphora and type logical grammar. Springer: Dordrecht.

Jarema, Gonia and Gary Libben (eds.). 2007. The mental lexicon: core perspectives. Amsterdam: Elsevier.

Johnson, Mark and Sam Bayer. 1995. Features and agreement in Lambek Categorial Grammar. In Proceedings of the Formal Grammar Workshop. Barcelona: FOLLI. 123-137.

Kamp, Hans and Uwe Reyle. 2011. Discourse representation theory. In Maienborn et al. (2011, 872-923).

Kracht, Marcus. 2002. On the semantics of locatives. Linguistics \& Philosophy 25. 57-132.

Kracht, Marcus. 2004. Against the Feature Bundle Theory of Case. In E. Brandner and H. Zinsmeister (eds.) New perspectives on case theory. Stanford, CA: CSLI Publications. 165-190.

Kracht, Marcus. 2008. The fine structure of spatial expressions. In Asbury et al. (2008, 35$62)$.

Kratzer, Angelika. 2007. Situations in natural language semantics. In E. N. Zalta (ed.) The Stanford encyclopedia of philosophy (Spring 2007 edition). Center for the Study of Language and Information, Stanford University: Stanford, CA.

Krifka, Manfred. 1998. The origins of telicity. In S. Rothstein (ed.) Events and grammar. Dordrecht: Kluwer. 187-235.

Krifka, Manfred. 2001. For a structured meaning account of questions and answers. In C. Féry and W. Sternefeld (eds.) Audiatur Vox Sapientiae. A Festschrift for Arnim von Stechow. Berlin: Akademie-Verlag. 287-319. 
Landman, Fred. 1991. Structures for semantics. Dordrecht: Kluwer.

Landman, Fred. 2000. Events and plurality: The Jerusalem lectures. Dordrecht: Kluwer.

LeStrade, Sander, Helen de Hoop and Kees de Schepper. 2010. Special issue on spatial case. Linguistics 48.

Levelt, Willem J. M. 1989. Speaking: From intention to articulation. Cambridge, MA: MIT Press.

Levinson, Stephen C. and David Wilkins (eds.). 2006. Grammars of space: Explorations in cognitive diversity. Cambridge: Cambridge University Press.

Maienborn, Claudia, Klaus von Heusinger and Paul Portner (eds.). 2011. Semantics: An international handbook of natural language meaning. Volume 1. Walter de Gruyter: Berlin \& Boston.

Maienborn, Claudia, Klaus von Heusinger and Paul Portner (eds.). 2012. Semantics: An international handbook of natural language meaning. Volume 2. Walter de Gruyter: Berlin \& Boston.

Merchant, Jason. 2001. The syntax of silence. Oxford: Oxford University Press.

Merchant, Jason. 2004. Fragments and ellipsis. Linguistics and Philosophy 27. 661-738.

Moortgat, Michael J. 2010. Typelogical grammar. In E. N. Zalta (ed.) The Stanford encyclopedia of philosophy. Stanford, CA: Center for the Study of Language and Information, Stanford University.

Moortgat, Michael J. 2011. Categorial type logics. In J. van Benthem and A. ter Meulen (eds.) Handbook of logic and language. Amsterdam \& Cambridge, MA: Elsevier \& MIT Press. 95-179.

Morrill, Glynn. 2011. Categorial grammar: Logical syntax, semantics, and processing. Oxford: Oxford University Press.

Nam, Senghou. 1995. The semantics of locative prepositional phrases in English. Doctoral dissertation. UCLA.

Napoli, Donna Jo and Joel Nevis. 1987. Inflected prepositions in Italian. Phonology 4. 195-209.

Pantcheva, Marina. 2008. The syntactic structure of locations, goals, and sources. Linguistics 48. 1043-1083.

Phillips, Colin. 2006. The real-time status of island phenomena. Language 82. 795-823.

Riemsdijk, Henk van and Riny Huybregts. 2007. Location and locality. In M. van Oostendorp and E. Anagnostopoulou (eds.) Progress in grammar: Articles at the 20th anniversary of the Comparison of Grammatical Models Group in Tilburg. Amsterdam: Meertens Instituut. 123-140.

Ritter, Elizabeth. 1991. Two functional categories in noun phrases: Evidence from Modern Hebrew. In S. Rothstein (ed.) Syntax and semantics 2. New York: Academic Press. $37-62$.

Ritter, Elizabeth. 1993. Where's gender? Linguistic Inqui 24. 795-803.

Rizzi, Luigi. 1988. Il sintagma preposizionale. In L. Renzi (ed.) Grande grammatica italiana di consultazione. Vol. 3. Bologna: Il Mulino. 497-530.

Romeu, Juan Fernandez. 2014. Cartografía mínima de las constucciones espaciales. Doctoral dissertation. Universidad Complutense de Madrid. 
Rothstein, Susan. 2004. Structuring events. Malden, MA \& Oxford: Blackwell.

Rothstein, Susan. 2008a. Telicity and atomicity. In Rothstein (2008b, 43-ó-78).

Rothstein, Susan (ed.). 2008b. Theoretical and crosslinguistic approaches to the semantics of aspects. Amsterdam \& Philadelphia: John Benjamins.

Schwarzschild, Roger. 1996. Pluralities. Dordrecht: Kluwer.

Svenonius, Peter. 2006. The emergence of axial parts. Nordlyd Troms $\varnothing$ Working Papers on Language \& Linguistics 33. 1-22.

Svenonius, Peter. 2008. Projections of P. In Asbury et al. (2008, 63-84).

Svenonius, Peter. 2010. Spatial P in English. In Cinque \& Rizzi (2010, 70-101).

Szabolcsi, Anna. 2010. Quantification. Cambridge: Cambridge University Press.

Talmy, Leonard. 2000. Toward a cognitive semantics. Vol. 1: Concept structuring systems; Vol. 2: Typology and process in concept structuring. Cambridge, MA: MIT Press.

Terzi, Arhonto. 2010. Locative prepositions and place. In Cinque \& Rizzi (2010, 196-224).

Thomas, Emma. 2001. On the expression of directional movement in English. Essex Graduate Student Papers in Language and Linguistics 4. 87-104.

Thomas, Emma. 2003. Manner-specificity as a factor in the acceptance of in and on in directional contexts. Essex Graduate Student Papers in Language and Linguistics 5. $117-146$.

Thomas, Emma. 2004. 'Syntactic' vs. 'semantic' telicity: IN and ON. Belgian Journal of Linguistics 18. 145-166.

Tortora, Christina. 2005. The preposition's preposition in Italian: Evidence for boundedness of space. In R. Gess and E. Rubin (eds.) Theoretical and experimental approaches to Romance linguistics. Amsterdam \& Philadelphia: John Benjamins. 307327.

Tortora, Christina. 2006. On the aspect of space: The case of PLACE in Italian and Spanish. In N. Pennello and D. Pescarini (eds.) Atti dell'undicesima giornata di dialettologia. Padova: CNR. 50-69.

Tortora, Christina. 2008. Aspect inside PLACE PPs. In Asbury et al. (2008, 273-301).

Ursini, Francesco-Alessio. 2011. On the syntax and semantics of "ser" and "estar". Lingue \& Linguaggio 9. 57-87.

Ursini, Francesco-Alessio. 2013. On the syntax and semantics of spatial Ps in Spanish. Borealis: An international journal about Hispanic Linguistic 2. 117-166.

Ursini, Francesco-Alessio. 2015. Another look at preposition stranding. In B. Erman, P. Shaw, G. Melchers and P. Sudnkvis (eds.) A Festschrift for Nils-Lennart Johansson. Stockholm: Stockholm University Press. 319-343.

Ursini, Francesco-Alessio and Nobu Akagi. 2013a. Another look at spatial prepositions and the modification problem. Iberia 5. 38-84.

Ursini, Francesco-Alessio and Nobu Akagi. 2013b. On the distributed morphology and semantics of spatial Ps. In I.-J. Lee and U. Dolgormaa (eds.) Proceedings of the 15th Seoul International Conference on Generative Grammar (SICOGG 15). Seoul: Hankuk University Press. 447-468.

Wunderlich, Dieter. 1991. How do prepositional phrases fit into compositional syntax and semantics? Linguistics 29. 591-621. 
Zwarts, Joost. 2005. Prepositional aspect and the algebra of paths. Linguistics \& Philosophy 28. 699-740.

Zwarts, Joost. 2008. Aspects of a typology of direction. In Rothstein (2008b, 79-106).

Zwarts, Joost and Yoad Winter. 2000. Vector space semantics: a model-theoretic analysis of locative prepositions. Journal of Logic, Language and Information 9. 169-211. 